\title{
DIAGRAM NOTATION FOR THE DERIVATION OF HYPERBOLIC MOMENT SYSTEMS*
}

\author{
JULIAN KOELLERMEIER ${ }^{\dagger}$ AND YUWEI FAN ${ }^{\ddagger}$
}

\begin{abstract}
We propose a diagram notation for the derivation of hyperbolic moment models for the Boltzmann equation that yields a better understanding of the resulting moment systems. So far several hyperbolic moment models were presented, but their derivations are often very technical and there is little insight into the explicit form of the equations. In our diagram notation, each term in the moment equations can be explicitly tracked throughout the derivation process and whether the resulting moment system is hyperbolic can be easily observed from the diagram. We apply the diagram notation to derive existing moment models, including Grad's moment equations, hyperbolic moment equations, quadrature-based moment equations, and a new set of simplified hyperbolic moment equations that was rarely studied before. The differences in the derivation are easily explained in the diagram notation and the explicit form of the equations can be computed straightforwardly as opposed to existing frameworks.
\end{abstract}

Keywords. Kinetic theory; moment method; hyperbolicity; Grad; diagram notation.

AMS subject classifications. 35L02; 82C40; 35Q20; 76P05.

\section{Introduction}

In gas kinetic theory the moment method was proposed by Grad in 1949 [24] to derive macroscopic moment equations from the Boltzmann equation and Grad's 13 moment equations are the most well-known moment model. However, it was pointed out that Grad's 13 moment equations for one-dimensional flow are not globally hyperbolic but only hyperbolic around the equilibrium [24, 40]. In [8], further investigation showed that the equilibrium is on the boundary of the hyperbolicity region for the three-dimensional case. For a first-order quasi-linear convection equation, loss of hyperbolicity indicates the equation with Cauchy data is no longer well-posed even locally and the uniqueness of the solution is lost [3]. Hence, for a long time, loss of hyperbolicity became a main obstacle for the development of the moment method.

In the past decades, some research brought new hope for the problem. In [37], the author proposed a new moment method based on the maximum entropy principle. This method yields globally hyperbolic moment models but unfortunately, the models beyond the Navier-Stokes theory cannot be written in analytical form and the equilibrium is on the boundary of the realizability domain, which leads to the models' loss of efficiency in numerical simulation.

To obtain a hyperbolic model from Grad's moment system, the authors of [6] investigated the characteristic structure of the coefficient matrix of the moment system for the one-dimensional case and proposed a hyperbolic regularization by modifying the last order equation of the moment system to obtain the Hyperbolic Moment Equations (HME). This method was extended to the multi-dimensional case in two ways $[7,22]$. Shortly after that, in [30], a quadrature-based moment method was proposed by computing the integrals using a suitable quadrature rule instead of exact integration. This method also yields globally hyperbolic moment equations, called Quadrature-Based Moment Equations (QBME). It was extended to the multi-dimensional case in two

${ }^{*}$ Received: February 02, 2018; Accepted (in revised form): January 28, 2020. Communicated by Giovanni Russo.

${ }^{\dagger}$ Institut für Mathematik, Freie Universität Berlin, 14195 Berlin, Germany and School of Mathematical Sciences, Peking University, Beijing, P.R. China (koellermeier@zedat.fu-berlin.de).

${ }_{\ddagger}^{\ddagger}$ Department of Mathematics, Stanford University, Stanford, CA 94305, USA (ywfan@stanford.edu). 
ways $[21,31]$. These two hyperbolic regularizations have been extended to the more general case in $[9,21]$ based on a truncation and an operator projection perspective, respectively. Numerical simulations $[5,12,13,33]$ demonstrate the efficiency of these regularizations. In recent years, the hyperbolic regularization in $[6,21]$ has been applied to a lot of fields besides gas kinetic theory and microflow, including semiconductor device simulation [10], plasma simulation [14,19], density functional theory [11], quantum gas kinetic theory [17] and rarefied relativistic Boltzmann equation [36]. However, the complexity of the regularized moment models limited their further application and the theoretical and numerical comparison between the regularizations in $[6,7]$ and [30] is not rich. In particular the derivation gives little insight into the explicit form of the equations.

In this paper, we propose a diagram notation to derive the hyperbolic moment models. In the diagram notation, each term in the ansatz and the equations is denoted by a node and each operator, for example the time-derivative operator, is denoted by one or several lines. In this notation, each term of the equations is represented by a path (from one node corresponding to a term in the ansatz to another node corresponding to another term in the equations) in the diagram. With the help of the diagram notation, the derivation of the moment system is explicit, concise and clear, so it yields a better understanding of the resulting moment systems. Moreover, it is easy to observe in the diagram whether the resulting system is hyperbolic. The diagram notation is applied to Grad's moment system, HME and QBME. We show that these three systems differ in their choice of discarding nodes in the diagram. The diagram notation also leads to the system of Simplified Hyperbolic Moment Equations (SHME), first mentioned in [34]. Due to the lack of results for this model in the literature, we study and compare the SHME to Grad's moment system, HME, and QBME in a numerical simulation of the 1D shock tube problem. Particularly, we study the behavior of SHME for increasing number of moments and conclude that its accuracy is not sufficient.

The rest of this paper is organized as follows: Motivational examples explaining the diagram notation are presented in Section 2. The necessary notation from kinetic theory and the standard derivation of moment models are briefly explained in Section 3 including a review of the existing hyperbolic models. Section 4 introduces the diagrambased derivation, which uses special diagrams that visualize every step of the derivation of the moment model. Existing moment models are derived using the new diagram notation in this section. For the SHME model, explicit equations, their properties, as well as numerical simulations addressing accuracy, are given in Appendix A.

\section{Motivation of diagram notation}

The use of the diagram notation can be motivated with the help of a simple but at the same time general equation to give an outline of the diagram framework without defining too much notation and also in order to show the applicability to a wider range of equations in the context of hyperbolic PDEs.

In the context of spectral methods, especially for linear, hyperbolic PDEs, there are several applications with a possibly large dimension due to the addition of another parameter or variable to the phase space. The reason for this can be the physical application itself or mathematical procedures such as Uncertainty Quantification (UQ), where a parameter of the PDE is treated as a possible independent variable to assess the impact of the parameter on the solution of the PDE $[25,41,45]$.

Throughout this paper, we will describe the one-dimensional spatial case, $x \in \mathbb{R}$, we will discuss and outline the case $x \in \mathbb{R}^{n}, n>1$ briefly in Section 4.9.

To motivate our diagram notation, we consider the following linear transport 
equation as example

$$
\partial_{t} f+c \cdot \partial_{x} f=0, \text { for } f=f(t, x, c) .
$$

We want to use a special spectral method to resolve the dependency of $c$ for the numerical solution of the equation. The following procedure will be explained using a generalized form of the linear transport Equation (2.1) because its treatment is most similar to the hyperbolic moment models in the next sections.

REMARK 2.1. The derivation of the model can also be performed for the wave equation in the form $\partial_{t t} u-c^{2} \cdot \partial_{x x} u=0$, for $u=u(t, x, c)$. However, the relation to hyperbolicity is more complicated. A non-linear ansatz requires the use of the form $\left(\partial_{t}+c \partial_{x}\right)\left(\partial_{t}-c \partial_{x}\right) u=0$ in order to show hyperbolicity. Similar complications arise for other second-order equations like the Klein-Gordon equation or the telegraph equation, see $[47]$.

We consider the following linear, hyperbolic PDE

$$
\partial_{t} f(t, x, c)+p(c) \cdot \partial_{x} f(t, x, c)=0, \text { for polynomial } p(c) .
$$

As ansatz for the unknown solution $f$ we use the following expansion in the variable $c$

$$
f(t, x, c)=\sum_{i=0}^{M} f_{i}(t, x) \Phi_{i}(c),
$$

where $M \in \mathbb{N}$ is the order of the expansion, $f_{i}$ are coefficients of the solution and $\Phi_{i}$ are basis functions, for $i=0, \ldots, M$, respectively. For the basis functions $\Phi_{i}$ we only assume that they are weighted orthonormal polynomials of degree $i$ in the variable $c$. The specific definitions of the basis functions are not important here and may depend on the parameter space (e.g., Hermite polynomials for $c \in \mathbb{R}$ or Legendre polynomials for $c \in[0,1]$ and respective weight functions).

We note that every orthogonal polynomial satisfies a three-term recurrence relation [1]. For the weighted Hermite polynomials, this reads

$$
c \Phi_{i}(c)=\alpha_{i} \Phi_{i-1}(c)+\beta_{i} \Phi_{i+1}(c),
$$

for some $\alpha_{i}, \beta_{i}, i \in \mathbb{N}$, depending on the scaling of the polynomial. The exact values of $\alpha_{i}, \beta_{i}$ are not important here. Without loss of generality, we will use the recursion in Equation (2.4) for our derivations, other recursion formulas can be treated analogously.

Now we consider three cases:

Case 1: $p(c)=c$

Case 2: $p(c)=c^{2}$

Case 3: $p(c)=c+c^{2}$

Case 1: $p(c)=c$

Inserting the ansatz (2.3) into (2.2) and using the recursion formula, we arrive at

$$
\sum_{i=0}^{M}\left(\Phi_{i}(c) \partial_{t} f_{i}+\alpha_{i} \Phi_{i-1}(c) \partial_{x} f_{i}+\beta_{i} \Phi_{i+1}(c) \partial_{x} f_{i}\right)=0
$$

The use of the recursion formula for each term can be represented in a diagram, see Figure 2.1, which shows how each basis function $\Phi_{i}(c)$ times $c$ is transformed into two 

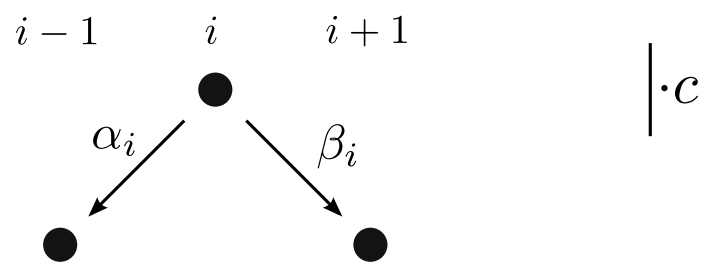

Fig. 2.1: Diagram for $p(c)=c$.

terms containing the basis functions $\Phi_{i-1}(c)$ and $\Phi_{i+1}(c)$ with their respective prefactors $\alpha_{i}$ and $\beta_{i}$ (written along the paths). Adding both paths leads to the spatial derivative summands in (2.5).

The resulting single $\operatorname{PDE}(2.5)$ is tested with the orthonormal test functions $\Phi_{j}$, $i=0, \ldots, M$ and then integrated over $c$ to get a system of equations of the form

$$
\partial_{t} f+\mathbf{A}_{c} \partial_{x} f=0
$$

for unknown vector $f=\left(f_{0}, \ldots, f_{M}\right)^{T} \in \mathbb{R}^{M+1}$ and system matrix $\mathbf{A}_{c} \in \mathbb{R}^{(M+1) \times(M+1)}$ defined by

$$
\mathbf{A}_{c}=\left(\begin{array}{ccccc}
0 & \beta_{0} & 0 & \ldots & 0 \\
\alpha_{1} & & \ddots & \ddots & \vdots \\
0 & \ddots & \ddots & \ddots & 0 \\
\vdots & \ddots & \ddots & & \beta_{M-1} \\
0 & \ldots & 0 & \alpha_{M} & 0
\end{array}\right)
$$

The eigenvalues of $\mathbf{A}_{c}$ can be easily computed and we obtain

$$
\lambda \in \sigma\left(\mathbf{A}_{c}\right) \Leftrightarrow \Phi_{M+1}(\lambda)=0,
$$

i.e. the eigenvalues of $\mathbf{A}_{c}$ are the real roots of the basis polynomial $\Phi_{M+1}$ with degree $M+1$. The system is thus hyperbolic. However, it is only that simple in this first case, as we will see in the following.

Case 2: $p(c)=c^{2}$

Inserting the ansatz (2.3) into (2.2) and using the recursion formula twice, we get

$$
\sum_{i=0}^{M}\left(\Phi_{i}(c) \partial_{t} f_{i}+\left(\alpha_{i} \alpha_{i-1} \Phi_{i-2}(c)+\alpha_{i} \beta_{i-1} \Phi_{i}(c)+\beta_{i} \alpha_{i+1} \Phi_{i}(c)+\beta_{i} \beta_{i+1} \Phi_{i+2}(c)\right) \partial_{x} f_{i}\right)=0
$$

The subsequent use of the recursion formula can again be represented in the diagram notation, see Figure 2.2. Here, the respective prefactors are multiplied along the way of the paths as can be seen by comparison with Equation (2.9) and all paths are added in the end to lead to the spatial derivative terms in (2.9).

After testing and integrating (2.9) the resulting PDE system reads

$$
\partial_{t} f+\mathbf{A}_{c^{2}} \partial_{x} f=0
$$




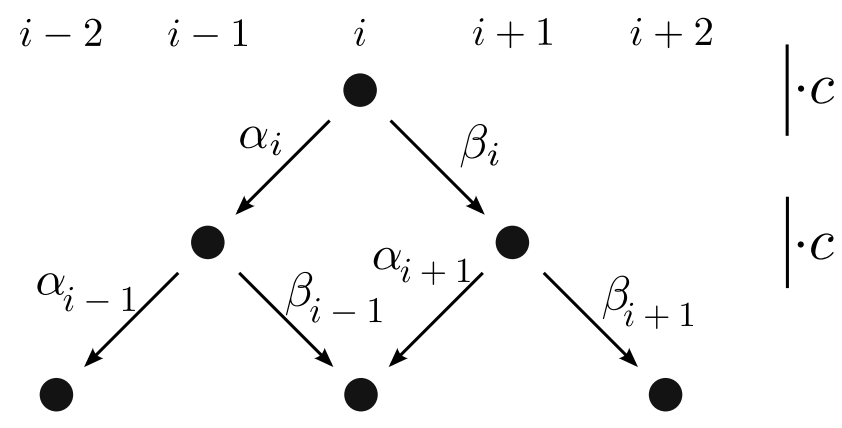

Fig. 2.2: Diagram for $p(c)=c^{2}$.

for system matrix $\mathbf{A}_{c^{2}} \in \mathbb{R}^{(M+1) \times(M+1)}$ defined by

$$
\mathbf{A}_{c^{2}}=\left(\begin{array}{cccccc}
\beta_{0} \alpha_{1} & 0 & \beta_{0} \beta_{1} & 0 & \ldots & 0 \\
0 & \alpha_{1} \beta_{0}+\beta_{1} \alpha_{2} & & \ddots & \ddots & \vdots \\
\alpha_{2} \alpha_{1} & & \ddots & \ddots & \ddots & 0 \\
0 & \ddots & \ddots & \ddots & & \beta_{M-2} \beta_{M-1} \\
\vdots & \ddots & \ddots & & \ddots & 0 \\
0 & \ldots & 0 & \alpha_{M-1} \alpha_{M} & 0 & \alpha_{M+1} \beta_{M+\beta_{M-1}} \alpha_{M}
\end{array}\right)
$$

However, the eigenvalues of $\mathbf{A}_{c}$ are not as simple as in the previous case. Specifically, we have

$$
\lambda \in \sigma\left(\mathbf{A}_{c}\right) \nRightarrow \Phi_{M+1}\left(\lambda^{2}\right)=0,
$$

i.e. the eigenvalues of $\mathbf{A}_{c}$ are not the squared real roots of the basis polynomial $\Phi_{M+1}$ with degree $M+1$, even though the roots are real and the system is hyperbolic.

This lack of structure is caused by the contribution of the polynomial with degree $M+1$ during the derivation and can be mitigated by applying a cut-off in the diagram notation as seen in Figure 2.3.

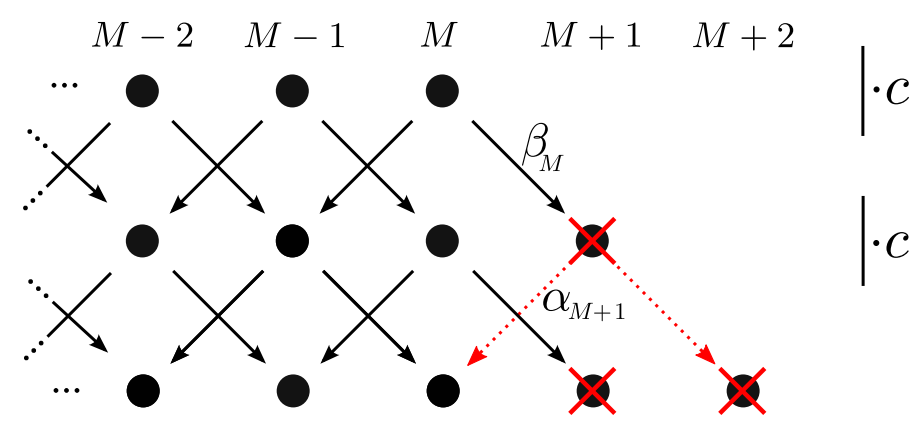

Fig. 2.3: Diagram for $p(c)=c^{2}$ with cut-off.

The cut-off eliminates the contribution of the polynomial with degree $M+1$, i.e. $\alpha_{M+1} \beta_{M}$, changing the last entry of the matrix. The modified system matrix is then 
given by

$$
\widetilde{\mathbf{A}}_{c^{2}}=\left(\begin{array}{cccccc}
\beta_{0} \alpha_{1} & 0 & \beta_{0} \beta_{1} & 0 & \ldots & 0 \\
0 & \alpha_{1} \beta_{0}+\beta_{1} \alpha_{2} & & \ddots & \ddots & \vdots \\
\alpha_{2} \alpha_{1} & & \ddots & \ddots & \ddots & 0 \\
0 & \ddots & \ddots & \ddots & & \beta_{M}-2 \beta_{M-1} \\
\vdots & \ddots & \ddots & & \ddots & 0 \\
0 & \ldots & 0 & \alpha_{M-1} \alpha_{M} & 0 & \beta_{M-1} \alpha_{M}
\end{array}\right) .
$$

We see that $\widetilde{\mathbf{A}}_{c^{2}}=\mathbf{A}_{c} \cdot \mathbf{A}_{c}=p\left(\mathbf{A}_{c}\right)$ and then the eigenvalues of $\widetilde{\mathbf{A}}_{c^{2}}$ are again simply evaluations of the polynomial of degree $M+1$

$$
\lambda \in \sigma\left(\widetilde{\mathbf{A}}_{c^{2}}\right) \Leftrightarrow \Phi_{M+1}\left(\lambda^{2}\right)=0
$$

Case 3: $p(c)=c+c^{2}=c \cdot(1+c)$

Combining both derivations from the previous cases, it is not difficult to see that the system matrix for this case is in fact the sum of the two previous matrices, i.e.

$$
\partial_{t} f+\mathbf{A}_{c+c^{2}} \partial_{x} f=0
$$

with system matrix $\mathbf{A}_{c+c^{2}}=\mathbf{A}_{c}+\mathbf{A}_{c^{2}}$

This time there is no structure in the eigenvalues due to the additional term from the polynomial with degree $M+1$. The exact same cut-off in the diagram solves this problem at the expense of a change in the last entry of the matrix corresponding to the previous example.

We can see that the modified matrix is given by $\widetilde{\mathbf{A}}_{c+c^{2}}=\mathbf{A}_{c}+\widetilde{\mathbf{A}}_{c^{2}}=\mathbf{A}_{c}+\mathbf{A}_{c} \cdot \mathbf{A}_{c}=p\left(\mathbf{A}_{c}\right)$. The new eigenvalues of the resulting matrix $\widetilde{\mathbf{A}}_{c+c^{2}}$ are then given by

$$
\lambda \in \sigma\left(\widetilde{\mathbf{A}}_{c+c^{2}}\right) \Leftrightarrow \Phi_{M+1}\left(\lambda+\lambda^{2}\right)=0,
$$

which leads to a hyperbolic system using the system matrix $\widetilde{\mathbf{A}}_{c+c^{2}}$.

It seems that the diagram notation is an easy way to depict the derivation and the changes required to obtain a particular eigenvalue structure in the motivational examples shown above. In these examples the success of the diagram notation is based on the fact that the matrix $\mathbf{A}_{c^{2}}$ needs to be represented as $\mathbf{A}_{c^{2}}=\mathbf{A}_{c} \cdot \mathbf{A}_{c}$, which goes back to the operator projection framework [21]. Each multiplication with $c$ needs to be cut-off or projected to the lower dimensional space separately.

In the following sections, we will extend the notation of the diagram to the more difficult case of the Boltzmann equation used for moment models that use a very nonlinear ansatz in comparison to (2.3). We will start by recalling the moment method for the Boltzmann equation.

\section{Moment method for the Boltzmann equation}

The Boltzmann transport equation describes the motion of particles by the evolution of the mass density function $f(t, x, c)$. The one-dimensional version of the Boltzmann equation reads

$$
\frac{\partial}{\partial t} f(t, x, c)+c \frac{\partial}{\partial x} f(t, x, c)=S(f)
$$


with position $x \in \mathbb{R}$ and microscopic velocity $c \in \mathbb{R}$. The right-hand side operator $S(f)$ models collisions, e.g., using the BGK collision operator [4]

$$
S(f)=\frac{1}{\tau}\left(f_{M}-f\right),
$$

with relaxation time $\tau \in \mathbb{R}$ and local Maxwellian $f_{M}(t, x, c)$ given by

$$
f_{M}(t, x, c)=\frac{\rho(t, x)}{\sqrt{2 \pi \theta(t, x)}} \exp \left(-\frac{(c-u(t, x))^{2}}{2 \theta(t, x)}\right) .
$$

Here the macroscopic quantities density $\rho(t, x)$, velocity $u(t, x)$ and temperature $\theta(t, x)$ are moments of the distribution function $f(t, x, c)$ in velocity space.

$$
\begin{aligned}
\rho(t, x) & =\int_{\mathbb{R}} f(t, x, c) \mathrm{d} c, \\
\rho(t, x) u(t, x) & =\int_{\mathbb{R}} c f(t, x, c) \mathrm{d} c, \\
\rho(t, x) \theta(t, x) & =\int_{\mathbb{R}}|c-u|^{2} f(t, x, c) \mathrm{d} c .
\end{aligned}
$$

Macroscopic conservation laws for mass, momentum and energy like the Euler equations can be derived by multiplication of Equation (3.1) with monomials in $c$ and integration over the velocity space, see e.g., [44].

Non-equilibrium effects occurring in rarefied gases cannot be described by simple models like the Euler equations [42]. Instead, moment methods can be applied to enlarge the number of unknowns and derive PDEs for the evolution of the additional variables. Grad's ansatz for the distribution function is an expansion around local equilibrium using a series of Hermite basis functions and yields the following expression for the distribution function $[21,24]$

$$
f(t, x, c)=\sum_{\alpha \in \mathbb{N}} f_{\alpha}(t, x) \mathcal{H}_{\alpha}^{[u(t, x), \theta(t, x)]}(c),
$$

with expansion coefficients $f_{\alpha}(t, x), \alpha \in \mathbb{N}$, and weighted Hermite functions $\mathcal{H}_{\alpha}^{[u, \theta]}$ defined by

$$
\mathcal{H}_{\alpha}^{[u, \theta]}(c)=(-1)^{\alpha} \frac{\mathrm{d}^{\alpha}}{\mathrm{d} c^{\alpha}} \omega^{[u, \theta]}(c), \quad \alpha \geq 0, \quad \omega^{[u, \theta]}(c)=\frac{1}{\sqrt{2 \pi \theta}} \exp \left(-\frac{(c-u)^{2}}{2 \theta}\right) .
$$

The properties of the weighted Hermite polynomials are listed in Appendix B. Note that expansion (3.7) is more complex than (2.3), because the ansatz explicitly includes moments of $f$ like $u, \theta$.

Definition (3.8) leads to the following constraints for the first three coefficients due to orthogonality of the basis functions, see (B.5),

$$
f_{0}=\rho, \quad f_{1}=f_{2}=0 .
$$

Substitution of the expansion (3.7) into (3.1) and application of suitable derivative (B.7) and recurrence relations (B.6) for the Hermite polynomials leads to explicit PDEs for the evolution of the coefficients $f_{\alpha}(t, x)$ by matching coefficients. Alternatively, the equations can be projected onto Hermite test functions via integration over velocity 
space, more details can be found in [6]. The final moment system has the following form according to $[21]$

$$
\begin{aligned}
& \frac{\partial f_{\alpha}}{\partial t}+\theta \frac{\partial f_{\alpha-1}}{\partial x}+u \frac{\partial f_{\alpha}}{\partial x}+(\alpha+1) \frac{\partial f_{\alpha+1}}{\partial x}+f_{\alpha-1} \frac{\partial u}{\partial t}+\left(\theta f_{\alpha-2}+u f_{\alpha-1}+(\alpha+1) f_{\alpha}\right) \frac{\partial u}{\partial x} \\
& +\frac{f_{\alpha-2}}{2} \frac{\partial \theta}{\partial t}+\frac{1}{2}\left(\theta f_{\alpha-3}+u f_{\alpha-2}+(\alpha+1) f_{\alpha-1}\right) \frac{\partial \theta}{\partial x}=S_{\alpha}, \quad \alpha \geq 3
\end{aligned}
$$

where the term $S_{\alpha}$ can be derived by the same expansion of the substituted collision term $S(f)$.

Due to the constraints (3.9), a finite set of equations can be obtained by using only $M+1$ variables $\boldsymbol{w}_{M}=\left(\rho, u, \theta, f_{3}, f_{4}, \ldots, f_{M}\right) \in \mathbb{R}^{M+1}$ and setting all other occurring terms in (3.10) to zero. After some simplifications the moment system can then be formulated in compact notation as

$$
\frac{\partial \boldsymbol{w}_{M}}{\partial t}+\mathbf{A} \frac{\partial \boldsymbol{w}_{M}}{\partial x}=\boldsymbol{S}
$$

where the matrix $\mathbf{A} \in \mathbb{R}^{(M+1) \times(M+1)}$ can be computed using (3.10) and the vector $\boldsymbol{S} \in \mathbb{R}^{M+1}$ contains the corresponding right-hand side terms in ascending order of the index $\alpha$. We refer to [6] or [33] for further details.

The expressions in Equation (3.10) make clear, that this moment method has many more terms than a standard (global) discretization using Grad's method using constant $(u, \theta)=\left(u_{0}, \theta_{0}\right)$. The benefits of this version using locally shifted and scaled basis functions as in (3.8) are explained in detail in [30].

REMARK 3.1. Note that the subsequent analysis of the moment system can be readily extended to a more general kinetic equation of the form $\partial_{t} f+p(v(c)) \partial_{x} f=S(f)$ for $v(c)$ a function of $c$ and $p(\cdot)$ a polynomial. The same holds for the multi-dimensional case $\partial_{t} f+\sum_{d=1}^{D} p_{d}(v(c)) \partial_{x_{d}} f=S(f)$ for which $x, c \in \mathbb{R}^{n}$. This also includes the radiative transfer equation. Details about these extensions can be found in [21]. We focus on the form (3.1) in order to present a concise derivation here.

3.1. Brief review of hyperbolic moment models. Grad's system (3.11) is only conditionally hyperbolic, as has been shown in $[6,8,33]$. The characteristic polynomial and thus the eigenvalues of the system matrix $\mathbf{A}$ depend on the highest coefficients which leads to complex eigenvalues and a lack of hyperbolicity already for moderate non-equilibrium states. This can cause the breakdown of numerical simulations [32] and renders Grad's system inappropriate for applications.

Several new hyperbolic models have been derived based on Grad's ansatz. In [21] a systematic approach for the derivation of hyperbolic moment models is given and the HME model [6,8], as well as the QBME model in [30], are examples for this approach. The derivation of the new hyperbolic moment models is based on subsequent projections of the equation during the derivation. This leads to changes in the last equations of the model hierarchies that suffice to render the models globally hyperbolic.

However, the derivation using projections is very theoretical and does not give direct insight into the explicit terms of the equation. It is therefore necessary to provide a more understandable method to derive the hyperbolic moment models in order to facilitate further development of existing models and derivation of new models.

In this paper, we want to show an alternative approach for the derivation of moment models in general and hyperbolic moment models in particular by means of the diagram notation described in the next section. 
REMARK 3.2. We note that only the left-hand side of (3.11) is important for the hyperbolicity and its structure is determined by the non-linearity of the basis functions. The right-hand side collision operator should not be changed due to its conservation properties. The collision operator especially plays an important role in numerical simulations later and when analyzing the stability of the methods, see e.g., $[18,46]$. More difficult collision operators can readily be used for simulations without changing the diagram framework.

\section{Diagram-based derivation}

The derivation of the hyperbolic moment models briefly described in Section 3.1 was based on two different methods so far. One is the original derivation of HME in $[6,8]$ and QBME in [30], which is a straightforward approach to derive hyperbolic models but is very complicated. The other one is the operator projection framework detailed in [21] and seems rather technical at first sight. Both methods have in common that there is only little insight into the explicit forms of the equations as they merely act as black box approaches. A third and more comprehensive way of deriving hyperbolic moment models is with the help of a special diagram notation that allows to visualize the deduction for further insight and variation of the models. The diagram-based method will furthermore lead to another hyperbolic moment model, the so-called Simplified Hyperbolic Moment Equations (SHME).

We start by recalling the derivation of Grad's method and use the newly developed diagram notation to depict the different steps exemplarily for each term.

4.1. Preliminaries. We define the transformed velocity variable

$$
\xi=\frac{c-u}{\sqrt{\theta}}
$$

then the weighted Hermite function can be denoted by

$$
\phi_{\alpha}^{[\theta]}(\xi):=\mathcal{H}_{\alpha}^{[u, \theta]}(c)=\frac{1}{\sqrt{2 \pi}} \theta^{-\frac{\alpha+1}{2}} \operatorname{He}_{\alpha}(\xi) \exp \left(-\frac{\xi^{2}}{2}\right)
$$

and Grad's expansion (3.7) is reformulated as

$$
f(t, x, c)=\sum_{\alpha=0}^{M} f_{\alpha}(t, x) \phi_{\alpha}^{[\theta]}(\xi) .
$$

Similar to the notation in [21], the superscript means that the basis functions $\phi_{\alpha}^{[\theta]}(\xi)$ depend on the macroscopic quantity $\theta(t, x)$. To simplify notation, we omit the superscript $\theta$ hereafter.

The derivation of the moment equations in general form now needs the computation of the terms in the Boltzmann Equation (3.1). The terms $\partial_{t} f$ and $\partial_{x} f$ are computed in the following way: for $s=x, t$ and for each term $f_{\alpha} \phi_{\alpha}$ in (4.3)

$$
\begin{aligned}
\partial_{s}\left(f_{\alpha} \phi_{\alpha}(\xi)\right) & =\partial_{s} f_{\alpha} \phi_{\alpha}(\xi)+f_{\alpha} \partial_{s} \phi_{\alpha}(\xi) \\
& =\frac{\partial_{s} f_{\alpha} \phi_{\alpha}(\xi)}{P_{2 a}}+\frac{f_{\alpha} \partial_{\theta} \phi_{\alpha}(\xi) \partial_{s} \theta}{P_{2 b}}+\frac{f_{\alpha} \partial_{\xi} \phi_{\alpha}(\xi) \partial_{s} \xi}{P_{12}},
\end{aligned}
$$

where the important adaptivity [28] is ensured by the last part

$$
\partial_{s} \phi_{\alpha}(\xi)=\partial_{\theta} \phi_{\alpha}(\xi) \partial_{s} \theta+\partial_{\xi} \phi_{\alpha}(\xi) \partial_{s} \xi
$$


The definition of the transformed velocity (4.1) indicates

$$
\partial_{s} \xi=-\frac{1}{\sqrt{\theta}} \partial_{s} u-\frac{\xi}{2 \theta} \partial_{s} \theta
$$

Here the symbols $P_{12}, P_{2 a}$ and $P_{2 b}$ in (4.4) are operators defined by

$$
\begin{aligned}
P_{12}\left(f_{\alpha} \phi_{\alpha}\right) & =P_{2}\left(P_{1}\left(f_{\alpha} \phi_{\alpha}\right)\right), \\
P_{1}\left(f_{\alpha} \phi_{\alpha}\right) & =P_{1}^{f}\left(f_{\alpha}\right) \cdot P_{1}^{\phi}\left(\phi_{\alpha}\right)=f_{\alpha} \cdot \partial_{\xi} \phi_{\alpha}(\xi), \\
P_{2}\left(f_{\alpha} \phi_{\alpha}\right) & =P_{2}^{f}\left(f_{\alpha}\right) \cdot P_{2}^{\phi}\left(\phi_{\alpha}\right)=f_{\alpha} \cdot \phi_{\alpha}(\xi) \partial_{s} \xi, \\
P_{2 a}\left(f_{\alpha} \phi_{\alpha}\right) & =P_{2 a}^{f}\left(f_{\alpha}\right) \cdot P_{2 a}^{\phi}\left(\phi_{\alpha}\right)=\partial_{s} f_{\alpha} \cdot \phi_{\alpha}(\xi), \\
P_{2 b}\left(f_{\alpha} \phi_{\alpha}\right) & =P_{2 b}^{f}\left(f_{\alpha}\right) \cdot P_{2 b}^{\phi}\left(\phi_{\alpha}\right)=f_{\alpha} \cdot \partial_{\theta} \phi_{\alpha}(\xi) \partial_{s} \theta .
\end{aligned}
$$

The multiplication of a distribution function with microscopic velocity $c \cdot f$ included in the term $c \cdot \partial_{x} f$ is straightforward and reads

$$
c \cdot f_{\alpha} \phi_{\alpha}(\xi)=(u+\sqrt{\theta} \xi) \cdot f_{\alpha} \phi_{\alpha}(\xi) .
$$

Similar as the definition of $P_{1}, P_{2}$, we define the operator $P_{3}$ as

$$
P_{3}\left(f_{\alpha} \phi_{\alpha}\right)=P_{3}^{f}\left(f_{\alpha}\right) \cdot P_{3}^{\phi}\left(\phi_{\alpha}\right)=f_{\alpha} \cdot(\sqrt{\theta} \xi+u) \phi_{\alpha}(\xi) .
$$

We remark here that all the operators $P_{1}, P_{2}, P_{2 a}, P_{2 b}$ and $P_{3}$ are linear operators.

From the computations above we can identify two necessary parts to deduce the moment system

(1) the derivative with respect to $t$ and $x$ : $\partial_{s} f$, for $s=t, x$ in (4.4),

(2) the multiplication with the microscopic velocity $c: c \cdot f$ in (4.12).

We note that the second part can also include multiplication by any polynomial function $p(c)$ as outlined in Section 2.

The definition of the operators $P_{1}, P_{2}, P_{2 a}$ and $P_{2 b}$ (4.8), (4.9), (4.10) and (4.11) indicate

$$
\partial_{s}\left(f_{\alpha} \phi_{\alpha}\right)=P_{2 a}\left(f_{\alpha} \phi_{\alpha}\right)+P_{2 b}\left(f_{\alpha} \phi_{\alpha}\right)+P_{2}\left(P_{1}\left(f_{\alpha} \phi_{\alpha}\right)\right)
$$

and the definition of the operator $P_{3}$ (4.13) indicates

$$
c \cdot f_{\alpha} \phi_{\alpha}=P_{3}\left(f_{\alpha} \phi_{\alpha}\right) \text {. }
$$

Here we point out that the critical difference to previous approaches like in $[6,21]$ is the additional subdivision into more than just the two combined steps (4.4) and (4.12). The specific form of the operators defined above will be made clear, once the basis functions are chosen.

Before the introduction of the diagram notation, we list some properties of the basis functions $\phi_{\alpha}(\xi)$. More details are provided in the Appendix B.

- $\xi$ derivative:

$$
\frac{\partial}{\partial \xi} \phi_{\alpha}(\xi)=-\sqrt{\theta} \phi_{\alpha+1}(\xi)
$$

- $\theta$ derivative:

$$
\frac{\partial}{\partial \theta} \phi_{\alpha}(\xi)=-\frac{\alpha+1}{2 \theta} \phi_{\alpha}(\xi)
$$

- multiplication with $\xi$ :

$$
\xi \phi_{\alpha}(\xi)=\sqrt{\theta} \phi_{\alpha+1}(\xi)+\frac{\alpha}{\sqrt{\theta}} \phi_{\alpha-1}(\xi) .
$$


4.2. Diagram-based framework. In this subsection, we introduce the diagram notation and prepare the tools for studying the moment systems.

First we introduce the four base elements of the diagram notation: node, serial number of the node, arrow and label of the arrow (see Figure 4.1 for samples). We take Figure $4.1 \mathrm{~b}$ as example. There are two nodes in Figure 4.1b: the serial number of the top-left node is $\alpha$ and that of the botton-right node is $\alpha+1$. Each node represents an expression $g_{k} \phi_{k}$ with $k$ to be the serial number of the node. Due to the linearity of the operator, the value of $g_{k}$ for the top-left node does not matter, so we assume it is $f_{\alpha}$ without loss of generality. The value of $g_{\alpha+1}$ for the bottom-right node is determined by the label of the arrow, e.g., using multiplication. In our notation, the arrow must start from the top to bottom, and the label of the arrow determines the value of the end node of the arrow. In this example, $g_{\alpha+1}=a \cdot f_{\alpha}$ for the bottom-right node. To sum up, the serial number of the end point of the arrow determines the basis function $\phi_{\text {serial number of the end point }}$ and the label of the arrow determines the value $g_{k}$ of the end point $g_{k}=$ value of the start point $\times$ label of the arrow, i.e. if the start point denotes $f_{\alpha} \phi_{\alpha}$, the operator is

$$
P\left(f_{\alpha} \phi_{\alpha}\right)=\text { label of the arrow } \times f_{\alpha} \phi_{\text {serial number of the end point } .}
$$

The diagram in Figure $4.1 \mathrm{~b}$ thus corresponds to an operator $\tilde{P}_{1}$ with $\tilde{P}_{1}\left(f_{\alpha} \phi_{\alpha}\right)=a f_{\alpha} \phi_{\alpha+1}$.

$\alpha$

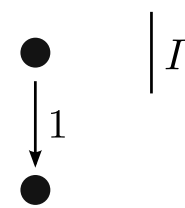

(a) $I\left(f_{\alpha} \phi_{\alpha}\right)=f_{\alpha} \phi_{\alpha}$

$\alpha-1 \quad \alpha \quad \alpha+1$

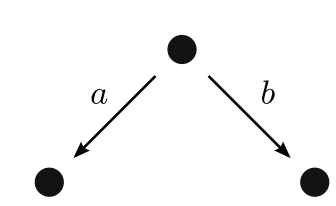

(c) $\tilde{P}_{2}\left(f_{\alpha} \phi_{\alpha}\right)=a f_{\alpha} \phi_{\alpha-1}+b f_{\alpha} \phi_{\alpha+1}$ $\alpha \quad \alpha+1$

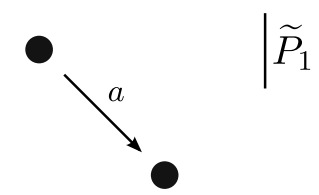

(b) $\tilde{P}_{1}\left(f_{\alpha} \phi_{\alpha}\right)=a f_{\alpha} \phi_{\alpha+1}$

$\alpha$

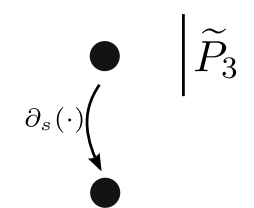

(d) $\tilde{P}_{3}\left(f_{\alpha} \phi_{\alpha}\right)=\partial_{s} f_{\alpha} \phi_{\alpha+1}$

Fig. 4.1: Samples of diagram notation for linear operators.

Here we show more examples to explain the diagram notation more clearly. In the diagram, the identity operator $I\left(f_{\alpha} \phi_{\alpha}\right)=f_{\alpha} \phi_{\alpha}$ is denoted by Figure 4.1a. In Figure 4.1c, there are two arrows. The meaning of each arrow has been defined above. Since the operator is a linear operator, the sum of two linear operators is also a linear operator, which indicates $\tilde{P}_{2}\left(f_{\alpha} \phi_{\alpha}\right)=a f_{\alpha} \phi_{\alpha-1}+b f_{\alpha} \phi_{\alpha+1}$. Instead of a value, the label of the arrow can also be a linear operator, which stands for applying the operator to the value of the start point. Thus, the diagram in Figure 4 .1d denotes $\tilde{P}_{3}\left(f_{\alpha} \phi_{\alpha}\right)=\partial_{s} f_{\alpha} \phi_{\alpha+1}$. We note that the bent arrow in Figure 4.1d is only a variation of the straight arrow and its purpose will be made clear later. With the definition of the four base elements, we 
$\alpha \quad \alpha+1$

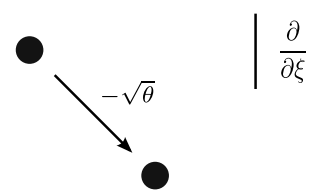

(a) $f_{\alpha} \frac{\partial}{\partial \xi} \phi_{\alpha}(\xi)=-\sqrt{\theta} f_{\alpha} \phi_{\alpha+1}(\xi)$ $\alpha$

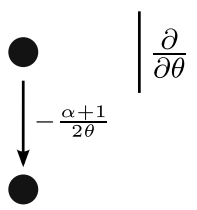

$\alpha-1 \quad \alpha \quad \alpha+1$

(b) $f_{\alpha} \partial_{\theta} \phi_{\alpha}(\xi)=-\frac{\alpha+1}{2 \theta} f_{\alpha} \phi_{\alpha}(\xi)$

(c) $f_{\alpha} \xi \phi_{\alpha}(\xi)=\sqrt{\theta} f_{\alpha} \phi_{\alpha+1}(\xi)+\frac{\alpha}{\sqrt{\theta}} f_{\alpha} \phi_{\alpha-1}(\xi)$

Fig. 4.2: Diagram of (4.16)-(4.18).

$\alpha \quad \alpha+1$

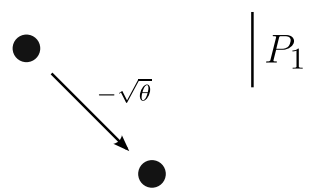

(a) $P_{1}$ as in (4.8)

$\alpha$

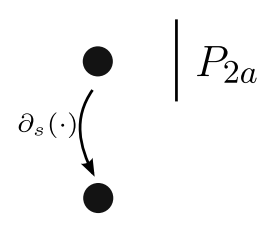

(c) $P_{2 a}$ as in $(4.10)$ $\alpha-1 \quad \alpha \quad \alpha+1$

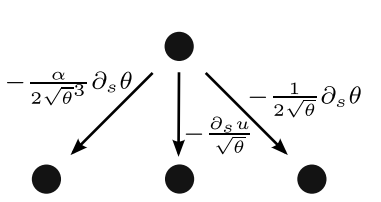

$\begin{array}{ccc}\alpha-1 & \alpha & \alpha+1\end{array}$

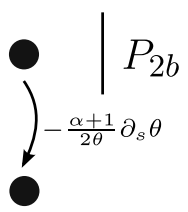

(d) $P_{2 b}$ as in (4.11) (b) $P_{2}$ as in (4.9)

$P_{2}$

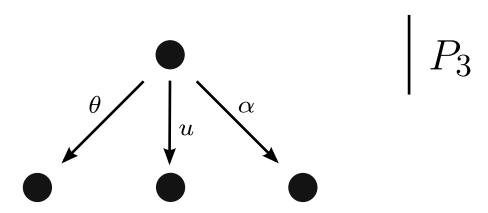

(e) $P_{3}$ as in (4.13)

Fig. 4.3: Diagram of the operators $P_{k}, k=1,2,3, P_{2 a}$ and $P_{2 b}$.

can directly denote the relations (4.16)-(4.18) by the diagram notation in Figures 4.2. These relations will be the building blocks of the diagram for the whole transformed Boltzmann equation. Next, we express the operators $P_{k}, k=1,2,3, P_{2 a}$ and $P_{2 b}$ in the diagram notation. Noticing (4.6), we have

$$
P_{2}\left(f_{\alpha} \phi_{\alpha}\right)=-\frac{\alpha}{2 \theta^{3 / 2}} \partial_{s} \theta f_{\alpha} \phi_{\alpha-1}-\frac{\partial_{s} u}{\sqrt{\theta}} f_{\alpha} \phi_{\alpha}-\frac{\partial_{s} \theta}{2 \sqrt{\theta}} f_{\alpha} \phi_{\alpha+1} .
$$

So the diagrams can be directly obtained from (4.8), (4.20), (4.10), (4.11) and (4.13). It is worth to remark that in the diagram of $P_{2 a}$ and $P_{2 b}$, both the arrows are straight top to bottom, so bent arrows are only used to distinguish these two operators. 
As all the building blocks for the derivation of the moment system are prepared, we can combine the blocks to represent composite operators. In fact, several operators can be applied in one step by simply adding up two diagrams as seen in the next figures. For simplification, from now on we omit the labels of the arrows for a better presentation. The labels can be seen in the previous Figures 4.3a-4.3e. The respective operator applied is always visible on the right side of the diagram.

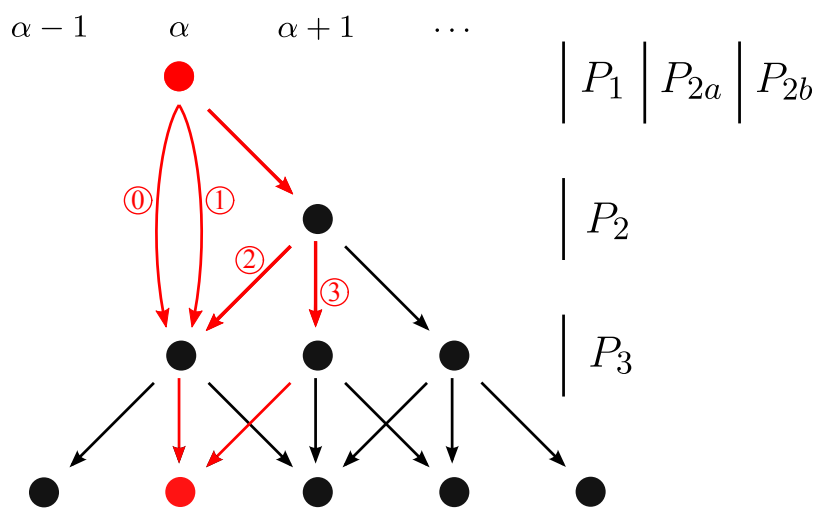

Fig. 4.4: Diagram notation for single basis function.

Now the operators corresponding to Figures 4.3a-4.3e have to be applied subsequently to compute the terms in Equations (4.4) and (4.12). If only a single basis function $f_{\alpha} \phi_{\alpha}(\xi)$ of fixed degree $\alpha$ was used as ansatz for the distribution function, i.e. $f(t, x, c)=f_{\alpha} \phi_{\alpha}(\xi)$, the diagram notation for the transport term $c \cdot \partial_{x} f$ in the Boltzmann equation would look as shown in Figure 4.4. The diagram in Figure 4.4 would then correspond to the computation of

$$
c \cdot \partial_{x} f=P_{3}\left(P_{2 a}\left(f_{\alpha} \phi_{\alpha}(\xi)\right)+P_{2 b}\left(f_{\alpha} \phi_{\alpha}\right)+P_{2}\left(P_{1}\left(f_{\alpha} \phi_{\alpha}(\xi)\right)\right)\right) .
$$

On the first level, the operators $P_{1}, P_{2 a}, P_{2 b}$ are applied and the result of $P_{1}$ will be applied to $P_{2}$ subsequently. As the results of $P_{2 a}, P_{2 b}$ and $P_{2}\left(P_{1}\right)$ have to be added, the curved arrows (corresponding to $P_{2 a}$ and $P_{2 b}$ ) skip one step and do not already end at the next level. Finally the operator $P_{3}$ depicts the multiplication with $c$.

According to Figure 4.4, the result in the bottom line of the figure includes five basis functions of degrees $\alpha-1, \alpha, \alpha+1, \alpha+2$ and $\alpha+3$ multiplied with the basis coefficient $f_{\alpha}$. Testing the result with orthogonal basis functions $\phi_{\beta}(\xi), \beta \in \mathbb{N}$, or equivalently matching coefficients of basis functions will lead to the appearance of coefficient $f_{\alpha}$ in the corresponding five equations.

The red paths in Figure 4.4 can be used to derive the contribution to the equation corresponding to $\phi_{\alpha}$ in the result as all coefficients in front of $\phi_{\alpha}$ are matched. There are four paths connecting the root node with the result node for equation $\alpha$. Each path is one term in the result and they have to be added in the end. Each one is computed starting from the root node and using the operators for $f_{\alpha} \phi_{\alpha}$ as follows

$$
\begin{array}{lll}
\text { (1): } f_{\alpha} \phi_{\alpha} & \stackrel{P_{2 a}(\zeta)}{-\rightarrow} \partial_{x} f_{\alpha} \phi_{\alpha} \stackrel{P_{3}(\downarrow)}{\rightarrow} u \partial_{x} f_{\alpha} \phi_{\alpha}, \\
\text { (1): } f_{\alpha} \phi_{\alpha} & \stackrel{P_{2 b}(\supsetneq)}{\rightarrow \rightarrow}-\frac{\alpha+1}{2 \theta} \partial_{x} \theta f_{\alpha} \phi_{\alpha} \stackrel{P_{3}(\downarrow)}{\rightarrow}\left(-\frac{\alpha+1}{2 \theta} \partial_{x} \theta\right) u f_{\alpha} \phi_{\alpha},
\end{array}
$$


(2): $f_{\alpha} \phi_{\alpha}$

$$
\begin{aligned}
& \underset{-\rightarrow}{P_{1}(\searrow)}-\sqrt{\theta} f_{\alpha} \phi_{\alpha+1} \\
& \underset{P_{2}(\swarrow)}{\longrightarrow}(-\sqrt{\theta})\left(-\frac{\alpha+1}{2 \sqrt{\theta}^{3}} \partial_{x} \theta\right) f_{\alpha} \phi_{\alpha} \\
& \underset{-\rightarrow}{P_{3}(\downarrow)}(-\sqrt{\theta})\left(-\frac{\alpha+1}{2 \sqrt{\theta}^{3}} \partial_{x} \theta\right) u f_{\alpha} \phi_{\alpha}, \\
& \underset{-\rightarrow}{P_{1}(\searrow)}-\sqrt{\theta} f_{\alpha} \phi_{\alpha+1} \\
& \stackrel{P_{2}(\downarrow)}{\rightarrow}(-\sqrt{\theta})\left(-\frac{\partial_{x} u}{\sqrt{\theta}}\right) f_{\alpha} \phi_{\alpha+1} \\
& \underset{-P_{3}(\swarrow)}{\longrightarrow}(-\sqrt{\theta})\left(-\frac{\partial_{x} u}{\sqrt{\theta}}\right)(\alpha+1) f_{\alpha} \phi_{\alpha},
\end{aligned}
$$

where only the red arrows in Figure 4.4 are included in the computation, as denoted by the small arrows $(\searrow, \swarrow, \downarrow, \supsetneq, \zeta)$ in brackets next to the operators. Summarized, the equation corresponding to testing with $\phi_{\alpha}$ will include the terms (1)+(1)+(2)+ (3), i.e.

$$
\begin{aligned}
& u \partial_{x} f_{\alpha}+\frac{\alpha+1}{2 \theta}\left(-\partial_{x} \theta u f_{\alpha}+\partial_{x} \theta u f_{\alpha}+2 \theta \partial_{x} u f_{\alpha}\right) \\
= & u \partial_{x} f_{\alpha}+(\alpha+1) \partial_{x} u f_{\alpha}
\end{aligned}
$$

Notice, that two terms cancel out in step (4.23). The expression in Equation (4.24) is exactly the same as the sum of terms in Equation (3.10) that correspond to spatial derivatives including the coefficient $f_{\alpha}$.

On the other hand, all entries in one equation can be obtained with the following procedure: After the application of all operators, the coefficients of basis functions are matched. This means that all coefficients in the result in front of one basis function of degree $\alpha$ are contributing to this equation. This is depicted by Figure 4.5 for the term $c \cdot \partial_{x} f$. The term $\partial_{t} f$ is obtained respectively without the last operator $P_{3}$.

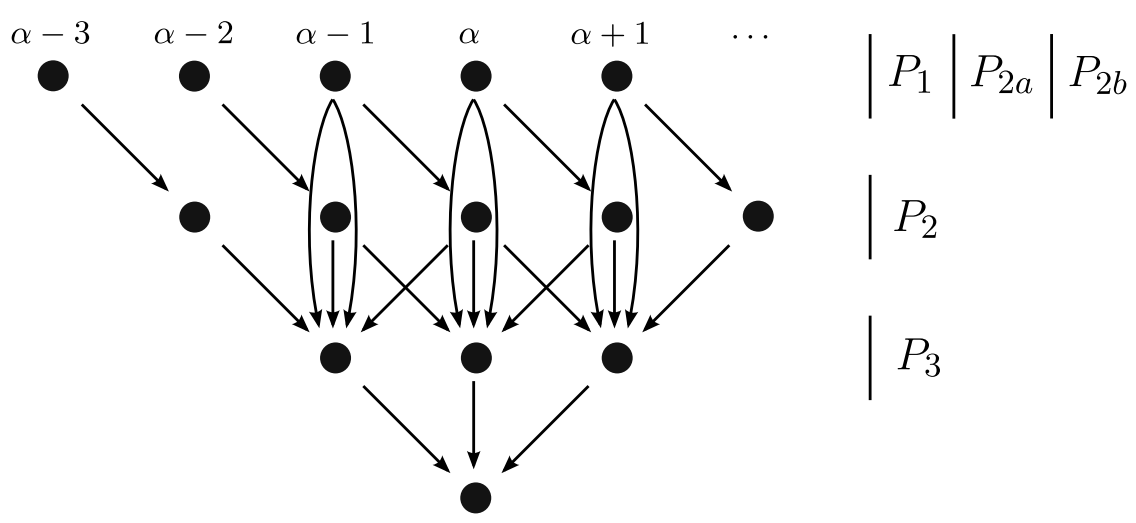

Fig. 4.5: notation for single equation.

Figure 4.5 contains all paths ending at the result node that is representing the basis function of degree $\alpha$. We see that equation $\alpha$ does only include the basis coefficients $f_{\alpha-3}, f_{\alpha-2}, f_{\alpha-1}, f_{\alpha}, f_{\alpha+1}$ in addition to the unknowns $u$ and $\theta$. 
Using the above notation all terms in the Boltzmann equation can be explicitly computed. Afterwards, a set of moment equations is obtained by matching the coefficients of basis functions. In the following, we explain the procedure for the previously derived moment systems in terms of the diagram notation.

4.3. Algorithmic description of diagram notation. The usage of the diagram notation can be explained in only a few steps, which algorithmically define the derivation of the resulting moment system. In the first phase, the respective diagram notation is created before the resulting moment system is derived in the second phase. The first phase reads as follows:

Phase 1: construction of diagram

(1) Use polynomial ansatz $f(t, x, c)=\sum_{\alpha} f_{\alpha}(t, x) \phi_{\alpha}(\xi)$ and transformed variable $\xi$

(2) Insert ansatz into BTE and use chain rule and transformation

(3) Define operators $P_{i}(\cdot)$ using recursion and derivative formulas

(4) Build diagram for single terms using operators

(5) Sum up single terms to whole system

After the definition of the ansatz and the corresponding basis functions, all other steps are straightforward and the result is the final diagram that can be used for the derivation of the moment system as explained in the next phase:

Phase 2: derivation of moment system

(1) Start with $M \in \mathbb{R}$ coefficients as root nodes of the diagram

(2) Identify paths to leafs of order $j \leq M$

(3) Optional: Discard cut-off terms for hyperbolicity following [21]

(4) Sum up contributions of single paths

(4.1) Start with root node of path

(4.2) Apply arrow labels along pathes

(4.3) Add result to respective equation $j$

The derivation thus only needs the number of moments and then follows an automated procedure that makes use of the previously constructed diagram. The result will be the moment equations. Examples are given in the following section.

For hyperbolicity, cutting off necessary terms in phase 2 step (3) is the crucial point and will be explained later. It requires to follow the operator framework in [21] during the cut-offs. See also Section 4.8 for the relation between hyperbolicity of moment models and the diagram notation.

Note that it is possible to use other types of kinetic equations instead of the Boltzmann transport equation (BTE) throughout the algorithm, and some examples can be found in [21] or by varying the polynomial $p(c)$ in Section 2. The only condition is that the ansatz has to be a weighted polynomial in the microscopic velocity variable $c$, such that a maximum entropy ansatz as in $[15,38]$ is not possible in the current setting. However, many of the moment methods in various applications are based on the expansion with a weighted polynomial basis, see for example $[17,23,36,43]$. An extension of the diagram notation towards maximum entropy models is an open question and we will focus on weighted polynomial expansions here.

4.4. Application: Grad's method. Grad's system is obtained by using the diagram notation without any modifications, cut-offs or projections during the derivation similar to the operator projection framework [21]. Only in the end, all the 
basis functions with degrees larger than $M$ are cut off. This will be denoted by red crosses in the following figures.

The time-derivative term $\partial_{t} f$ is computed according to Figure 4.6.

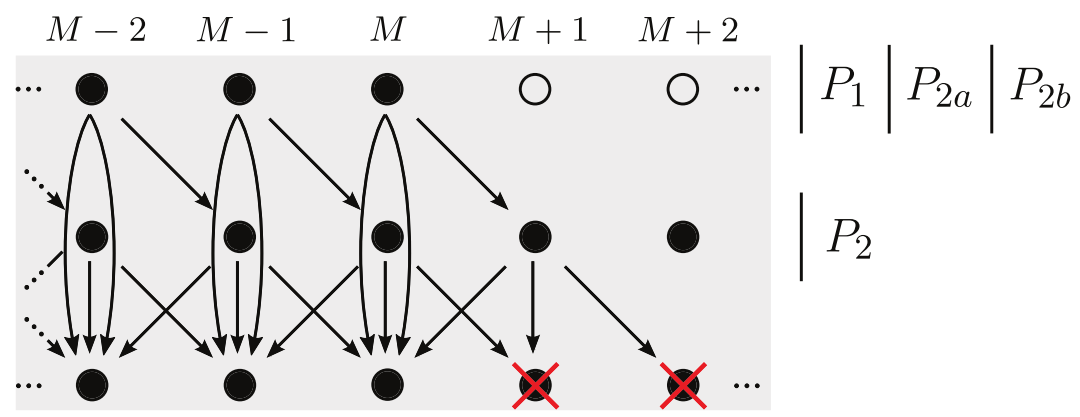

Fig. 4.6: Diagram for $\partial_{t} f$ in Grad's method.

Starting from expansion (4.3) using $M+1$ basis coefficients $f_{0}, \ldots, f_{M}$, the operators $P_{1}, P_{2}, P_{2 a}$ and $P_{2 b}$ are applied successively and the derivation follows the same steps as in the previous diagrams. After the last step the resulting basis functions with degrees $M+1, M+2, \ldots$ are set to zero corresponding to the usual cut-off of Grad's method.

The computation of the transport term $c \cdot \partial_{x} f$ is illustrated similarly in Figure 4.7. The only difference is the additional operator $P_{3}$, which corresponds to the multiplication with $c$. Only after the last step the resulting basis functions with degree $M+1, M+2, \ldots$ are cut-off. Grad's system can be obtained by formal summation of both diagram components.

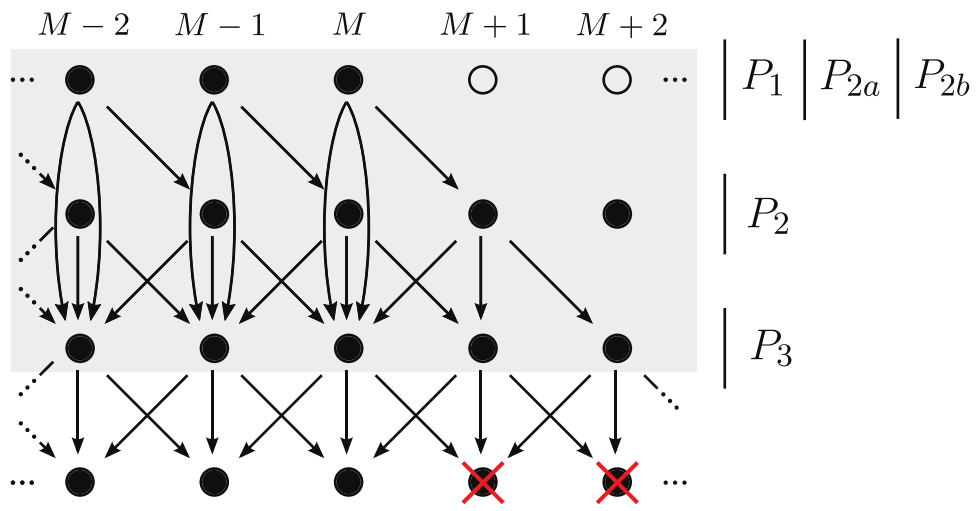

Fig. 4.7: Diagram for $c \cdot \partial_{x} f$ in Grad's method.

Note that there is no cut-off after the space derivative and thus the gray shaded space-derivative part of Figure 4.7 is treated differently than the time-derivative part in Figure 4.6. According to [21], this is the main reason for the lack of hyperbolicity of Grad's method.

4.5. Application: HME. During the derivation of Grad's method the treatments of the time derivative and the spatial derivative are different because there 
is no cut-off after the spatial derivative. This observation was already made in [9] and is one reason for the HME regularization explained in Section 3.1.

The diagram notation yields another viewpoint that allows for understanding the HME regularization. The computation of the term $\partial_{t} f$ is exactly the same as for Grad's method, see Figure 4.7. The transport term $c \cdot \partial_{x} f$, however, changes due to an additional cut-off after the operators $P_{1}, P_{2}, P_{2 a}$ and $P_{2 b}$ are applied. This is depicted in Figure 4.8. The additional cut-off (or projection as it is called in the frame of [21]) leads to a change in the last equation corresponding to basis function $\phi_{M}$ as seen by the red marking in the last step of the diagram. The other equations are kept unchanged and the small modification to the last equation suffices to render the system hyperbolic.

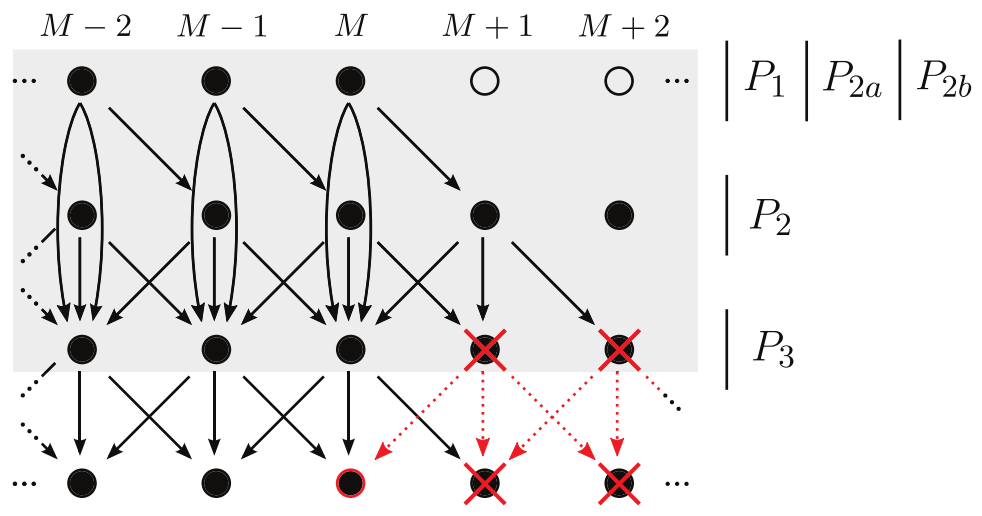

Fig. 4.8: Diagram for $c \cdot \partial_{x} f$ in $H M E$.

In contrast to Grad's method, the HME method uses the same treatment for the space and time derivatives as can be seen in the gray shaded areas comparing Figures 4.6 and 4.8. This guarantees hyperbolicity as shown in [21].

The differences in the last equation of the HME system can be computed following the paths leading to the $M$-th equation that were cut-off. Only two paths need to be taken into account. According to Figure 4.9, the two paths are computed in similar ways as the paths in Equation (4.22) as

$$
\begin{array}{rll}
\text { (1): } f_{M} \phi_{M} & \rightarrow & (M+1) \partial_{x} u f_{M} \phi_{M}, \text { equal to path (3) in (4.22), } \\
\text { (2): } f_{M-1} \phi_{M-1} \stackrel{P_{1}(\searrow)}{-\longrightarrow}-\sqrt{\theta} f_{M-1} \phi_{M} \\
P_{2}(\searrow) \\
\stackrel{\rightarrow}{\rightarrow}(-\sqrt{\theta})\left(-\frac{\partial_{x} \theta}{2 \sqrt{\theta}}\right) f_{M-1} \phi_{M+1} \\
P_{3}(\swarrow) \\
\stackrel{-\longrightarrow}{\rightarrow}(-\sqrt{\theta})\left(-\frac{\partial_{x} \theta}{2 \sqrt{\theta}}\right)(M+1) f_{M-1} \phi_{M} .
\end{array}
$$

By leaving out the two paths computed in (4.25), the last equation of the HME system thus gets an additional regularization term

$$
R_{M}^{H M E}=-(M+1) \partial_{x} u f_{M}-\frac{M+1}{2} \partial_{x} \theta f_{M-1} .
$$

4.6. Application: QBME. The QBME model can be written by a different modification of the previous diagram including one additional cut-off after the operator 


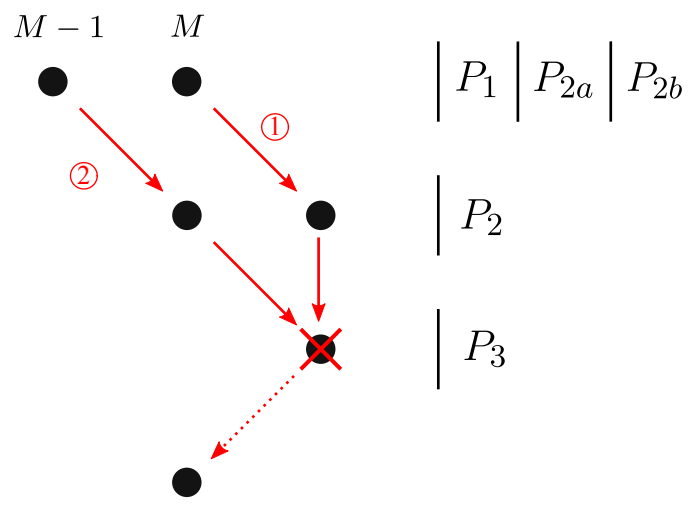

Fig. 4.9: Changes of term $c \cdot \partial_{x} f$ in $H M E$.

$P_{1}$. First, we consider the time-derivative term $\partial_{t} f$ of the Boltzmann equation, which can be derived in the diagram notation for QBME as shown in Figure 4.10. As opposed

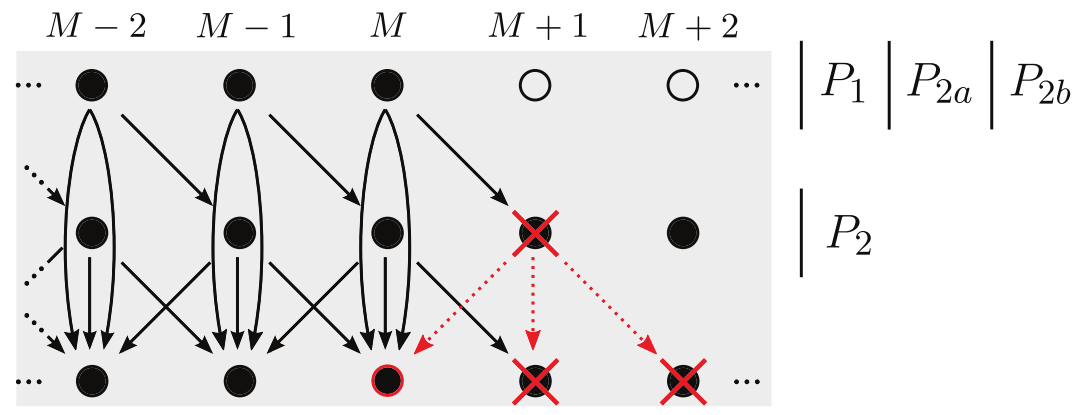

Fig. 4.10: Diagram for $\partial_{t} f$ in $Q B M E$.

to Grad's model and HME, the additional cut-off leads to a change in the last equation of the time-derivative term that can be computed similarly to the HME changes in Equation (4.25).

The transport term $c \cdot \partial_{x} f$ also uses this additional cut-off, which is consistent to the time-derivative term. Figure 4.11 visualizes the derivation. The early cut-off now leads to changes in the last two equations as can be seen by the red circles in the last line.

The cut-off of all terms including basis functions of degree $M+1$ leads to vanishing terms of degree $M+2$ as well and thus, similar as for HME, the same treatment of the time and space derivatives in Figures 4.10 and 4.11 ensures hyperbolicity of the QBME model.

The changed terms are computed using Figure 4.12a. The time-derivative change 


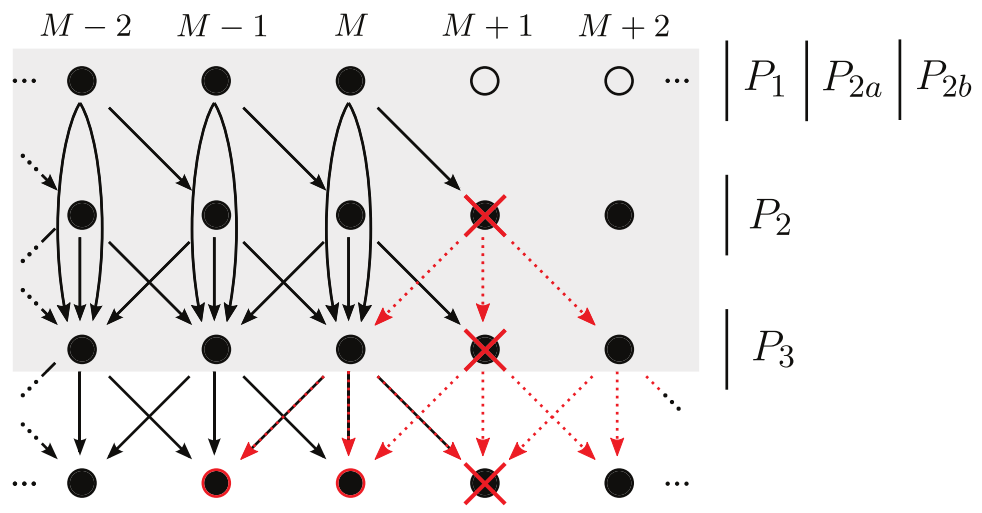

Fig. 4.11: Diagram for $c \cdot \partial_{x} f$ in $Q B M E$.

is derived as follows

$$
\text { (1): } \begin{aligned}
f_{M} \phi_{M} \stackrel{\substack{P_{2}(\searrow) \\
\rightarrow}}{\rightarrow}-\sqrt{\theta} f_{M} \phi_{M+1} \\
\stackrel{P_{2}(\swarrow)}{-\rightarrow}(-\sqrt{\theta})\left(\frac{M+1}{2 \sqrt{ }^{3}} \partial_{t} \theta\right) f_{M} \phi_{M} \\
=\frac{M+1}{2 \theta} \partial_{t} \theta f_{M} \phi_{M} .
\end{aligned}
$$

The changes in the transport term are derived using Figure 4.12b, respectively. The contributions are from both $f_{M-1}$ and $f_{M}$, where the term for $f_{M}$ is split up into three single paths. The terms read

(1): $f_{M-1} \phi_{M-1} \quad \rightarrow \frac{M+1}{2} \partial_{x} \theta f_{M-1} \phi_{M}$, equal to path (2) in (4.25),

(2a): $f_{M} \phi_{M} \quad \rightarrow(M+1) \partial_{x} u f_{M} \phi_{M}$, equal to path (1) in (4.25),

(2b): $f_{M} \phi_{M} \quad \rightarrow u \frac{M+1}{2 \theta} \partial_{x} \theta f_{M} \phi_{M}$, equal to path (2) in (4.22),

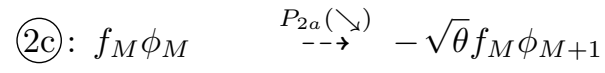

$$
\begin{aligned}
& \stackrel{P_{2}(\swarrow)}{--\rightarrow}(-\sqrt{\theta})\left(-\frac{M+1}{2 \sqrt{\theta}^{3}} \partial_{x} \theta\right) f_{M} \phi_{M} \\
& \stackrel{P_{3}(\swarrow)}{-\rightarrow}(-\sqrt{\theta})\left(-\frac{M+1}{2 \sqrt{\theta}^{3}} \partial_{x} \theta\right) M f_{M} \phi_{M-1} \\
& =\frac{M(M+1)}{2 \theta} \partial_{x} \theta f_{M} \phi_{M-1} .
\end{aligned}
$$

The regularization term in equation $M-1$ is thus given by

$$
R_{M-1}^{Q B M E}=-\frac{M(M+1)}{2 \theta} \partial_{x} \theta f_{M}
$$

and the sum of all terms added to equation $M$ in the QBME model reads

$$
R_{M}^{Q B M E}=-\frac{M+1}{2} \partial_{x} \theta f_{M-1}-(M+1) \partial_{x} u f_{M}-u \frac{M+1}{2 \theta} \partial_{x} \theta f_{M} .
$$




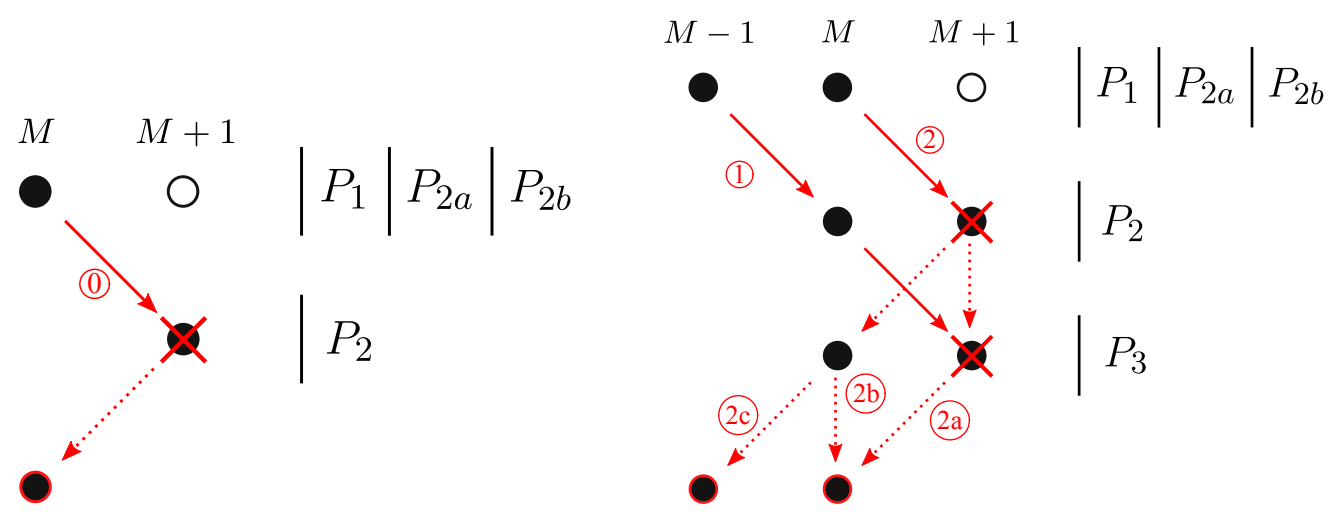

(a) $\partial_{t} f$

(b) $c \cdot \partial_{x} f$

Fig. 4.12: Changes of terms $\partial_{t} f$ 4.12a and $c \cdot \partial_{x} f$ 4.12b in QBME. Only the paths for changed equations are displayed.

4.7. Application: SHME. The transformation (4.1) that leads to Equations (4.4) and (4.12) allows for an efficient discretization on the one hand. However, the resulting system of equations becomes more difficult on the other hand as seen by the recursion formulas that include derivatives of $\theta$ and $u$. This introduces some nonlinearity into the equations. Unfortunately, the lack of hyperbolicity makes it necessary to regularize the equations so that some terms need to be neglected or cut off as derived in the previous sections. Following the ideas in [34] where mainly the five-moment case was described, another approach is to keep the simple form of the equation and use only a small part of the non-linear features of the fully transformed Boltzmann equation.

The non-linearity of the transformed Boltzmann equation enters the derivation at two different points. One is the transformation of the derivative $\partial_{s} f$ for $s=t, x$ in Equation (4.4) and the other is the multiplication with $c=u+\sqrt{\theta} \xi$ in Equation (4.12). The non-linearity in the multiplication is relatively easy to handle as it does not lead to additional derivatives of $u$ or $\theta$ due to the chain rule. The transformation of $\partial_{s} f$, however, includes $\partial_{s} u$ and $\partial_{s} \theta$, which lead to two almost full columns in the system matrix as we will see in the next section.

A straightforward modification is to neglect the non-linearity in the transformed derivative [34], which leads to simplified physics. It corresponds to setting

$$
\partial_{s}\left(f_{\alpha} \phi_{\alpha}(\xi)\right)=\partial_{s} f_{\alpha} \phi_{\alpha}(\xi)+f_{\alpha} \partial_{s} \phi_{\alpha}(\xi) \approx \partial_{s} f_{\alpha} \phi_{\alpha}(\xi)
$$

where the dependency of the basis function on the macroscopic variables $u$ and $\theta$ (and thus all terms $\partial_{s} u$ and $\partial_{s} \theta$ ) is neglected in (4.31) with respect to (4.4). The resulting moment system is called the Simplified Hyperbolic Moment Equations (SHME) in the following.

The macroscopic conservation laws of mass, momentum and energy must not be changed. The approximation in Equation (4.31) is therefore only applied to basis functions with polynomial degree $\alpha<2$.

The approximation in Equation (4.31) is equivalent to a cut-off of the terms produced by the operators $P_{1}, P_{2 b}$ and $P_{2}$, this can be seen in Figures 4.13 and 4.14 where the respective paths marked in red are cut off. Note that the term from $P_{2 a}$ is not neglected, according to (4.31). 


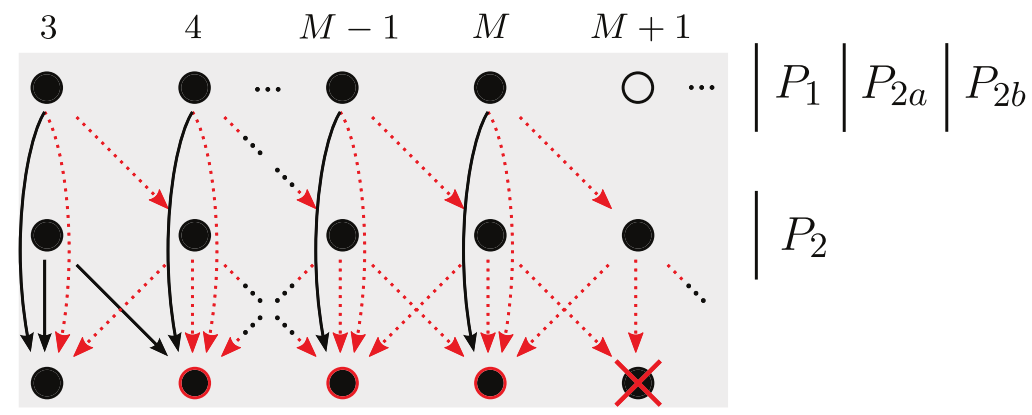

Fig. 4.13: Diagram for $\partial_{t} f$ in SHME.

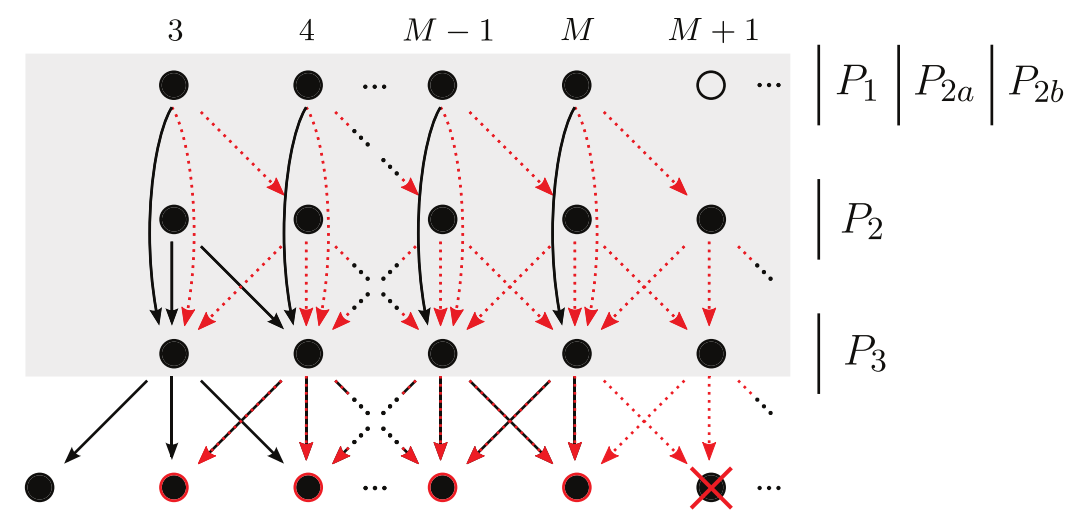

Fig. 4.14: Diagram for $c \cdot \partial_{x} f$ in SHME.

The time and space derivatives in the shaded areas of Figures 4.13 and 4.14 result in the same treatment and again indicate hyperbolicity of the SHME model as there is no contribution of terms coming from the basis function of degree $M+1$ after the derivative.

In Figure 4.13 it is important to note that the equation for $\alpha=3$ does not change, as both deleted paths ending at the result node for that equation already canceled out previously. This can be seen by the following computation

$$
\begin{aligned}
& \text { (1) } f_{3} \phi_{3} \stackrel{P_{2 b}(\supseteq)}{-\rightarrow}-\frac{4}{2 \theta} \partial_{t} \theta f_{3} \phi_{3} \text {, } \\
& \text { (2) } f_{3} \phi_{3} \stackrel{P_{1}(\searrow)}{\rightarrow}-\sqrt{\theta} f_{3} \phi_{4} \\
& \stackrel{P_{2}(\swarrow)}{-\rightarrow}(-\sqrt{\theta})\left(-\frac{4}{2 \sqrt{\theta}^{3}} \partial_{t} \theta\right) f_{3} \phi_{3} \\
& =\frac{4}{2 \theta} \partial_{t} \theta f_{3} \phi_{3} \text {. }
\end{aligned}
$$

From (4.32) it is clear that both terms cancel out so that the equation for $\alpha=3$ does not include any changes in the time-derivative term, in comparison of Figure 4.4 and the cancellation in Equation (4.23). The same holds true for the spatial derivative terms 
in the equation for $\alpha=2$ as seen in Figure 4.14. This means that the conservation laws are not changed and only higher-order equations include regularization terms.

With the help of the diagram notation, it is directly clear that the space and time derivatives are obviously treated the same and thus SHME is hyperbolic, as the condition for hyperbolicity from the operator projection framework is fulfilled [21]. In Appendix A we give a concise analysis of the resulting system. As expected, neglecting much of the non-linear physics leads to a loss of accuracy of the higher-order models, despite satisfying hyperbolicity.

\subsection{Relation between hyperbolicity and diagram notation.}

Hyperbolicity of the resulting equations can be shown with the help of the more theoretical framework in [21]. The actual derivation is then depicted by the diagram notation following the steps outlined by the operator projection framework.

As shown in [21] hyperbolicity requires the same treatment of the time-derivative term and the spatial derivative term in the Boltzmann Equation 3.1. For a resulting system of hyperbolic moment equations it is therefore paramount to apply the same cut-off to both terms in the Boltzmann equation during the derivation using the diagram notation. By comparison of the gray-shaded part of the diagrams for Grad, HME, QBME and SHME in Section 3 it can be seen that Grad's method does not fulfill the condition for hyperbolicity. On the other hand, the hyperbolic models HME, QBME and SHME use the same cut-off for the gray-shaded parts in the time-derivative term, as well as in the spatial derivative term. The same is true for motivational example in Section 2 , where each multiplication is cut-off separately as proven by operator projection in [21] and depicted by the diagrams. The hyperbolicity can thus easily be spotted by inspection of the diagrams. This is another beneficial property of the diagram notation. It is an easy tool to visualize not only the derivation but also the effect of the different cut-off strategies on the resulting equations and hyperbolicity, respectively.

4.9. Discussion. The diagram notation is a beneficial tool for the derivation of hyperbolic moment systems. It helps to understand the effects of the different regularizations and allows for a quick and simple computation of the regularization terms without a lot of difficult matrix notation involved. It furthermore opens up new possibilities for the derivation of other methods. The SHME system is only one example and many more are possible.

The new diagram notation can be seen as another language for the hyperbolic moment model reduction. The first framework [9] studied the hyperbolicity using functional algebra and PDE language, whereas the operator projection framework [21] was relying on operators and linear algebra language. The PDE language was the first rigorous statement, while the linear algebra language was very concise and abstract. The diagram notation will now provide the interested reader with the necessary hands-on intuition to understand the model derivation. Its existence is a justification to regularize Grad's moment model using one of the hyperbolic models HME, QBME, SHME.

As previously stated in Sections 3.1and 4.3, the diagram notation can also be applied to other equations similar to the projection procedure in [21], provided a polynomial ansatz is used. The choice of basis functions has to be made accordingly and the recursion and derivative formulas in Equations (4.16), (4.17) and (4.18) need to be computed from the properties of the basis function. However, as every set of orthogonal polynomials has a three-term recurrence relation and formulas for the derivatives, this extension is straightforward. One example of this is the recently developed shallow water moment model, where Legendre polynomials are used to allow for a vertical change of 
the velocity and similar problems with hyperbolicity occur, see [35]. Further results can be obtained by applying the notation to other moment models, e.g., [17, 20,26].

The multi-dimensional case can be done in a similar way, but we will not show the results in the scope of this paper. In general, the multi-dimensional case will use the description of the basis functions as spherical harmonics in tensor notation, see e.g., $[27,29]$. The available recursion formulas for the respective tensor formulation allow for a concise notation in applications for $2 \mathrm{D}$ or $3 \mathrm{D}$ velocity space.

\section{Conclusion}

We introduced a new way of deriving hyperbolic moment models by means of a diagram notation. The notation dissects the derivation process in small steps and makes it easy to spot differences between various existing models. All the ideas of the model reduction frameworks in $[9,21,30]$ are reflected in the new notation. The diagram notation is thus a new visualized and concise method to study derivation and hyperbolicity of moment systems.

We exemplified the derivation of the standard Grad model and the hyperbolic HME and QBME models using the new diagram notation. Additionally, we showed a general derivation of the Simplified Hyperbolic Moment Equations (SHME). The model was derived by neglecting large parts of the non-linearity during the derivation. Preliminary numerical results showed that despite the hyperbolicity of the model, its accuracy is not sufficient.

The diagram notation supplements the previous frameworks in analysing and deriving existing and new moment models. As opposed to the existing frameworks, it is a straightforward method to derive the explicit form of the resulting moment models.

Appendix A. SHME model equations and properties. We will briefly discuss the SHME system first mentioned in [34], analogously to [33] for HME and QBME.

Written in the form of Equation (3.11), the SHME system derived in Section 4.7 reads

$$
\mathbf{A}_{\text {SHME }}=\left(\begin{array}{ccccccccc}
u & \rho & & & & & & \\
\frac{\theta}{\rho} & u & 1 & & & & & \\
& 2 \theta & u & \frac{6}{\rho} & & & & \\
& 0 & \frac{\rho \theta}{2} & u & 4 & & & \\
0 & \vdots & 0 & \theta & u & 5 & & \\
\vdots & \vdots & \vdots & 0 & \ddots & \ddots & \ddots & \\
\vdots & \vdots & \vdots & \vdots & & \theta & u & M \\
0 & 0 & 0 & 0 & & & \theta & u
\end{array}\right)
$$

The red entries mark differences with respect to the standard Grad's method. A $_{\text {SHME }}$ is much simpler than all other hyperbolic models (i.e. HME and QBME) due to the many zero entries in the first four columns, which include the main non-linear parts.

The SHME system is hyperbolic, as the system matrix is the same as Grad's system matrix evaluated at equilibrium

$$
\mathbf{A}_{\mathrm{SHME}}\left(\boldsymbol{w}_{M}\right)=\mathbf{A}_{\mathrm{Grad}}\left(\boldsymbol{w}_{M}^{e q}\right)
$$

so it is the linearization of the matrix at equilibrium. The work in [18] indicates that SHME is also linearly stable around equilibrium and satisfies Yong's stability condition 
[46]. Due to (A.2), the characteristic polynomial of SHME is equal to the characteristic polynomial of Grad's system evaluated at equilibrium (compare [6])

$$
\chi_{M}(\lambda)=\theta^{\frac{M+1}{2}} \operatorname{He}_{M+1}\left(\frac{\lambda-u}{\sqrt{\theta}}\right) .
$$

The eigenvalues are the real roots of the corresponding Hermite polynomial, like for the HME and QBME models. This leads to a hyperbolic system. The eigenvalues still adaptively adjust to the flow, even though a lot of the non-linearity of the transformation was neglected during the derivation. The eigenvectors $\boldsymbol{v}_{k}$ corresponding to the eigenvalues $\lambda_{k}=u+c_{k} \sqrt{\theta}$ with $c_{k}$ the $k$-th root of $\mathrm{He}_{M+1}(c)$ are given by

$$
\begin{aligned}
& v_{k, 1}=\rho \mathrm{He}_{0}\left(c_{k}\right), \quad v_{k, 2}=\theta^{1 / 2} \mathrm{He}_{1}\left(c_{k}\right), \quad v_{k, 3}=\theta \mathrm{He}_{2}\left(c_{k}\right), \\
& v_{k, j}=\frac{1}{(j-1) !} \rho \theta^{(j-1) / 2} \mathrm{He}_{j-1}\left(c_{k}\right), \quad j=4, \cdots, M+1,
\end{aligned}
$$

equal to evaluating the eigenvalues of the HME model at equilibrium [6]. Direct calculations yield

$$
\nabla_{\boldsymbol{w}_{M}} \lambda_{k} \cdot \boldsymbol{v}_{k}=1 \cdot v_{k, 2}+\frac{c_{k}}{2 \sqrt{\theta}} \cdot v_{k, 3}=\frac{\sqrt{\theta}}{2} c_{k}\left(c_{k}^{2}+1\right),
$$

thus $\nabla_{\boldsymbol{w}_{M}} \lambda_{k} \cdot \boldsymbol{v}_{k}=0$ if and only if $c_{k}=0$, which indicates each characteristic field of the SHME is either genuinely non-linear or linearly degenerate. The properties of each kind of elementary wave can be studied following [6]. Since the first three equations of the SHME remain unchanged and the first three elements of the eigenvectors are the same as those of HME, most of the analysis in [6] holds for SHME.

The transformation of the SHME system to a different set of variables does not lead to a simpler system as is the case for HME and QBME [33]. Instead the system written in convective variables yields a full lower diagonal part of the system matrix.

Only the first three equations of the SHME remain unchanged and can thus still be written in conservative form, while the other equations cannot. In numerical simulations we expect larger differences between the SHME and Grad's method because of the large simplification of the SHME model. The number of entries that are changed also increases with increasing $M$ affecting convergence of the model. For smaller $M$, however, there are not too many differences also in comparison with QBME and HME and simulation results are expected to give satisfactory approximation quality.

A brief numerical comparison of the SHME model with respect to existing models is performed using the one-dimensional shock tube test case also used in [6] and [33]. The results can be seen as an extension of the first preliminary results in [34].

The initial conditions are chosen as

$$
\boldsymbol{w}_{M}(0, x)= \begin{cases}\boldsymbol{w}_{M}^{L}=(7,0,1,0, \ldots, 0)^{T} & \text { if } x<0, \\ \boldsymbol{w}_{M}^{R}=(1,0,1,0, \ldots, 0)^{T} & \text { if } x>0\end{cases}
$$

for variable vector $\boldsymbol{w}_{M}=\left(\rho, u, \theta, f_{3}, \ldots, f_{M}\right)^{T}, M \geq 4$. The non-linear relaxation time $\tau$ is chosen as $\tau=\frac{\mathrm{Kn}}{\rho}$, where we consider different Knudsen numbers $\mathrm{Kn}_{1}=0.05$ representing a small Knudsen number and $\mathrm{Kn}_{2}=0.5$ for a relatively large Knudsen number.

The computational domain $[-2,2]$ is discretized using 4000 cells and the following figures show the numerical solutions at end time $t_{\mathrm{END}}=0.3$ using constant $\Delta t=0.0001$ corresponding to a CFL number of ca. 0.45 . 
All numerical results are computed using the first-order, non-conservative PRICE scheme as described in [16] and also employed for the one-dimensional tests in [33], from which all other settings, e.g., for the computation of the non-conservative products, are also adopted. It was shown that these settings allow for stable and accurate solutions to the non-conservative moment systems. For details we refer to the aforementioned papers.

We recall the results from [34], and show solutions for $M=4$ and $M=9$, i.e. five or ten variables, respectively, and small Knudsen number $\mathrm{Kn}_{1}=0.05$.

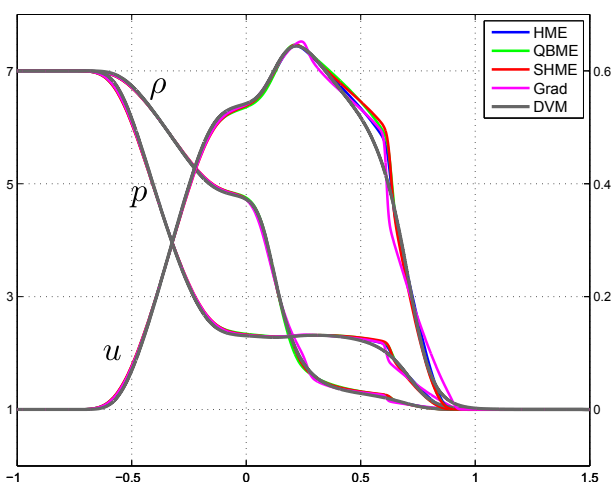

(a) $M=4$

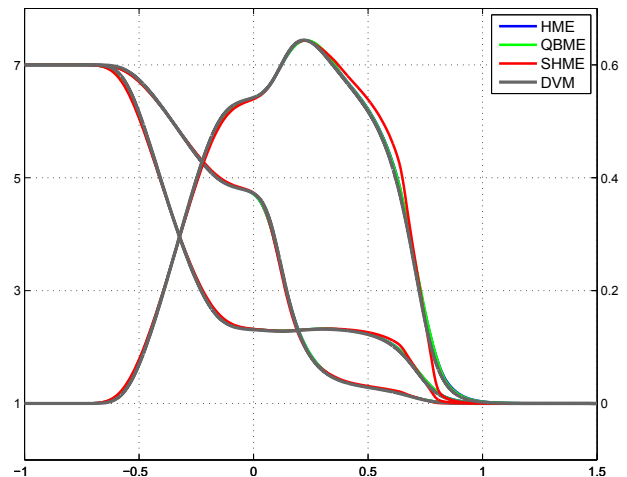

(b) $M=9$

Fig. A.1: Moment model comparison for Grad, HME, QBME, SHME and DVM reference solution, $K n=0.05$. The left $y$-axis is for $\rho$ and $p$, the right $y$-axis is for $u$, compare [34].

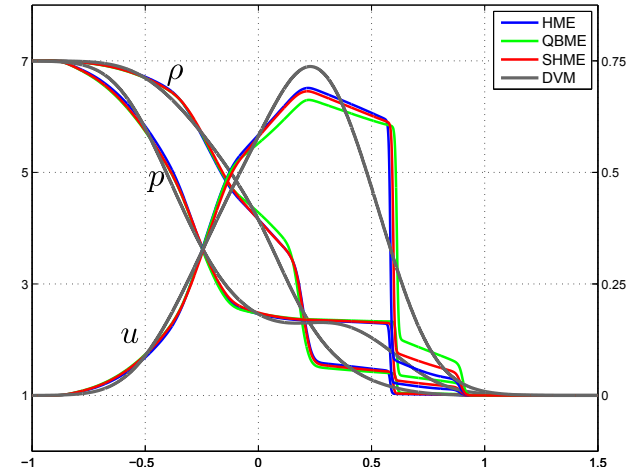

(a) $M=4$

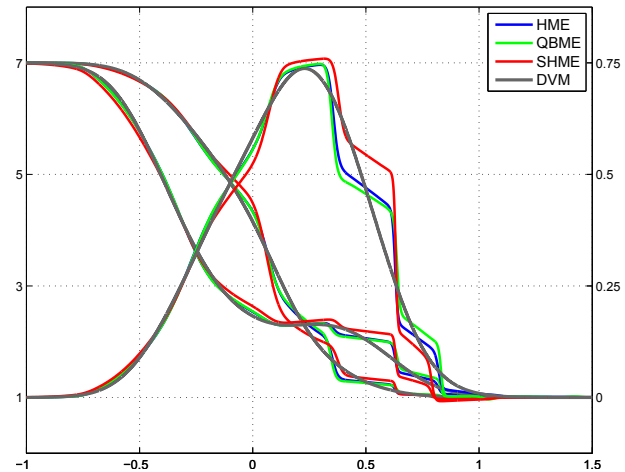

(b) $M=9$

Fig. A.2: Moment model comparison for Grad, HME, QBME, SHME and DVM reference solution, $K n=0.5$. The left $y$-axis is for $\rho$ and $p$, the right $y$-axis is for $u$, compare [34].

The results for the five-moment case $M=4$ in Figure A.1a show that the SHME model has similar accuracy as the other hyperbolic models HME and QBME and the deviation from the reference DVM solution (taken from [6]) is small. However, increasing the number of variables using $M=9$ in Figure A.1b does not lead to a very accurate SHME solution, whereas the other models converge to the reference solution. 
The results of all hyperbolic models for the larger Knudsen number $\mathrm{Kn}_{2}=0.5$ in Figure A.2 differ more from the reference solution due to stronger non-equilibrium effects caused by the larger Knudsen number. In addition, Grad's model loses hyperbolicity in this setup and fails to produce a solution. For $M=4$ in Figure A.2a the SHME results lie between the HME and the QBME model with comparable accuracy. For the larger $M=9$ in Figure A.2b differences are more obvious in the right half of the domain. The HME and QBME results are relatively close to each other whereas the SHME model differs substantially.

The convergence study in Figures A.3a and A.3b uses averaged solutions obtained by two subsequent values of $M$ in the same way as performed in [2] and the plots for HME and QBME are taken from [33]. The SHME model clearly fails to converge and

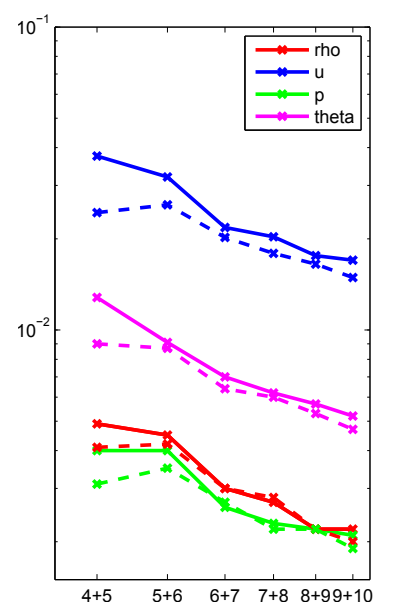

(a) QBME (solid) + HME (dashed)

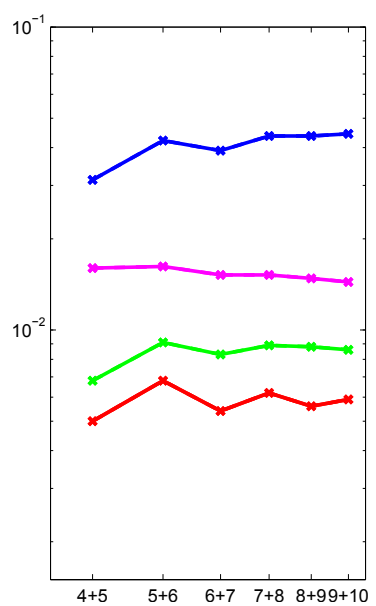

(b) SHME

Fig. A.3: Averaged solution convergence of HME and QBME and no convergence for SHME, for $K n=0.5$.

shows constant or even increasing errors with larger $M$. This can be attributed to the drastic simplification and large number of changes made in the system matrix in (A.1). It turns out that the neglected non-linearity, which was set to zero during the derivation of the SHME model, is in fact necessary for convergence of the solution in this test case.

Appendix B. Hermite polynomials and weighted Hermite functions. In this appendix, we list some properties of the Hermite polynomials and weighted Hermite functions without proof. Details can be found in $[1,22,39]$.

The generalized probabilists' Hermite polynomials $\mathrm{He}_{n}^{[u, \theta]}(c), \theta>0$ are defined as

$$
\mathrm{He}_{n}^{[u, \theta]}(c)=\frac{(-1)^{n}}{\omega^{[u, \theta]}(c)} \frac{\mathrm{d}^{n}}{\mathrm{~d} c^{n}} \omega^{[u, \theta]}(c), n \in \mathbb{N}, \quad \omega^{[u, \theta]}(c)=\frac{1}{\sqrt{2 \pi \theta}} \exp \left(-\frac{(c-u)^{2}}{2 \theta}\right),
$$

which are orthogonal with respect to the weight function $\omega^{[u, \theta]}(c)$, i.e.

$$
\int_{-\infty}^{+\infty} \operatorname{He}_{n}^{[u, \theta]}(c) \operatorname{He}_{m}^{[u, \theta]}(c) \omega^{[u, \theta]}(c) \mathrm{d} c=\frac{n !}{\theta^{n}} \delta_{m, n} \quad \forall m, n \in \mathbb{N} .
$$


The generalized Hermite polynomials satisfy the three-term recursion relation

$$
c \mathrm{He}_{n}^{[u, \theta]}(c)=\theta \mathrm{He}_{n+1}^{[u, \theta]}(c)+u \mathrm{He}_{n}^{[u, \theta]}(c)+n \mathrm{He}_{n-1}^{[u, \theta]}(c), \quad n \geq 1 .
$$

If we define weighted Hermite functions $\mathcal{H}_{n}^{[u, \theta]}(c)$ as

$$
\mathcal{H}_{n}^{[u, \theta]}(c)=\omega^{[u, \theta]}(c) \mathrm{He}_{n}^{[u, \theta]}(c),
$$

then these functions satisfy the orthogonality relation

$$
\int_{-\infty}^{\infty} \mathcal{H}_{n}^{[u, \theta]}(c) \mathcal{H}_{m}^{[u, \theta]}(c) / \omega^{[u, \theta]}(c) \mathrm{d} c=\frac{n !}{\theta^{n}} \delta_{n, m}, \quad \forall m, n \in \mathbb{N}
$$

and the three-term recursion relation

$$
c \mathcal{H}_{n}^{[u, \theta]}(c)=\theta \mathcal{H}_{n+1}^{[u, \theta]}(c)+u \mathcal{H}_{n}^{[u, \theta]}(c)+n \mathcal{H}_{n-1}^{[u, \theta]}(c), \quad n \geq 1,
$$

as well as the differential relation

$$
\mathrm{d} \mathcal{H}_{n}^{[u, \theta]}(c)=\mathcal{H}_{n+1}^{[u, \theta]}(c) \mathrm{d}(u-c)+\frac{1}{2} \mathcal{H}_{n+2}^{[u, \theta]}(c) \mathrm{d} \theta, \quad n \in \mathbb{N} .
$$

If $u=0$ and $\theta=1$, the generalized Hermite polynomials reduce to the classical Hermite polynomials, and we denote them by $\operatorname{He}_{n}(c)$.

If we let $\xi=\frac{c-u}{\theta}$ and define

$$
\phi_{n}^{[\theta]}(\xi):=\mathcal{H}_{n}^{[u, \theta]}(c)=\frac{1}{\sqrt{2 \pi}} \exp \left(-\frac{\xi^{2}}{2}\right) \mathrm{He}_{n}(\xi) \theta^{-(n+1) / 2},
$$

then $\phi^{[\theta]}$ is also orthogonal with respect to $\exp \left(\xi^{2} / 2\right)$ and satisfies the three-term recursion relation

$$
\xi \phi_{n}^{[\theta]}(\xi)=\sqrt{\theta} \phi_{n+1}^{[\theta]}(\xi)+\frac{n}{\sqrt{\theta}} \phi_{n-1}^{[\theta]}(\xi), \quad n \geq 1
$$

and the differential relation

$$
\mathrm{d} \phi_{n}^{[\theta]}(\xi)=-\sqrt{\theta} \phi_{n+1}^{[\theta]}(\xi) \mathrm{d} \xi-\frac{n+1}{2 \theta} \phi_{n}(\xi) \mathrm{d} \theta, \quad n \in \mathbb{N} .
$$

\section{REFERENCES}

[1] M. Abramowitz and I.A. Stegun, Handbook of Mathematical Functions: With Formulas, Graphs, and Mathematical Tables, Appl. Math. Series. Dover Publications, 32(1):239, 1965. 2, B

[2] J.D. Au, M. Torrilhon, and W. Weiss, The shock tube experiment in extended thermodynamics, Phys. Fluids, 13(8):2423-2432, 2001. A

[3] A.V. Azevedo, B. Marchesin, B.J. Plohr, and K. Zumbrun, Nonuniqueness of solutions of Riemann problems, Z. Angew. Math. Phys., 47(6):977-998, 1996. 1

[4] P.L. Bhatnagar, E.P. Gross, and M. Krook, A model for collision processes in gases. 1. Small amplitude processes in charged and neutral one-component systems, Phys. Rev., 94:511-525, 1954. 3

[5] Z. Cai, Numerical simulation of microflows with moment method, in T. Karayiannis, C.S. Konig, and S. Balabani (eds.), Proceedings of the 4th Micro and Nano Flow Conference 2014, Brunel University, ID218, 2014. 1

[6] Z. Cai, Y. Fan, and R. Li, Globally hyperbolic regularization of Grad's moment system in one dimensional space, Commun. Math. Sci., 11(2):547-571, 2013. 1, 3, 3, 3.1, 4, 4.1, A, A, A, A 
[7] Z. Cai, Y. Fan, and R. Li, Globally hyperbolic regularization of Grad's moment system, Commun. Pure Appl. Math., 67(3):464-518, 2014. 1

[8] Z. Cai, Y. Fan, and R. Li, On hyperbolicity of 13-moment system, Kinet. Relat. Models, 7(3):415432, 2014. 1, 3.1, 4

[9] Z. Cai, Y. Fan, and R. Li, A framework on moment model reduction for kinetic equation, SIAM J. Appl. Math., 75(5):2001-2023, 2015. 1, 4.5, 4.9, 5

[10] Z. Cai, Y. Fan, R. Li, T. Lu, and Y. Wang, Quantum hydrodynamic model by moment closure of Wigner equation, J. Math. Phys., 53(10):103503, 2012. 1

[11] Z. Cai, Y. Fan, R. Li, T. Lu, and W. Yao, Quantum hydrodynamic model of density functional theory, J. Math. Chem., 51(7):1747-1771, 2013. 1

[12] Z. Cai, Y. Fan, R. Li, and Z. Qiao, Dimension-reduced hyperbolic moment method for the Boltzmann equation with BGK-type collision, Commun. Comput. Phys., 15(5):1368-1406, 2014. 1

[13] Z. Cai, R. Li, and Z. Qiao, Globally hyperbolic regularized moment method with applications to microflow simulation, Comput. Fluids, 81:95-109, 2013. 1

[14] Z. Cai, R. Li, and Y. Wang, Solving Vlasov equation using NRxx method, SIAM J. Sci. Comput., 35(6):A2807-A2831, 2013. 1

[15] V. Camiola and V. Romano, 2DEG-3DEG charge transport model for MOSFET based on the maximum entropy principle, SIAM J. Appl. Math., 73(4):1439-1459, 2013. 4.3

[16] A. Canestrelli, A. Siviglia, M. Dumbser, and E.F. Toro, Well-balanced high-order centred schemes for non-conservative hyperbolic systems. Applications to shallow water equations with fixed and mobile bed, Adv. Water Resour., 32(6):834-844, 2009. A

[17] Y. Di, Y. Fan, and R. Li, 13-moment system with global hyperbolicity for quantum gas, J. Stat. Phys., 167(5):1280-1302, 2017. 1, 4.3, 4.9

[18] Y. Di, Y. Fan, R. Li, and L. Zheng, Linear stability of hyperbolic moment models for Boltzmann equation, Numer. Math. Theor. Meth. Appl., 10:255-277, 2017. 3.2, A

[19] Y. Di, Z. Kou, and R. Li, High order moment closure for Vlasov-Maxwell equations, Front. Math. China, 10(5):1087-1100, 2015. 1

[20] J. Duan, Y. Kuang, and H. Tang, Model reduction of a kinetic swarming model by operator projection, East Asian J. Appl. Math., 8(1):151-180, 2018. 4.9

[21] Y. Fan, J. Koellermeier, J. Li, R. Li, and M. Torrilhon, Model reduction of kinetic equations by operator projection, J. Stat. Phys., 162(2):457-486, 2016. 1, 2, 3, 3, 3.1, 3.1, 4, 4.1, 4.1, 4.3, $4.4,4.4,4.5,4.5,4.7,4.8,4.9,5$

[22] Y. Fan and R. Li, Globally hyperbolic moment system by generalized Hermite expansion, Sci. Sin. Math., 45(10):1635-1676, 2015. 1, B

[23] M. Frank, C. Hauck, and K. Küppers, Convergence of filtered spherical harmonic equations for radiation transport, Commun. Math. Sci., 14(5):1443-1465, 2016. 4.3

[24] H. Grad, On the kinetic theory of rarefied gases, Commun. Pure Appl. Math., 2(4):331-407, 1949. 1,3

[25] J. Hu and S. Jin, Uncertainty Quantification for Kinetic Equations, Springer International Publishing, Cham., 193-229, 2017. 2

[26] Z. Hu, R. Li, and Z. Qiao, Acceleration for microflow simulations of high-order moment models by using lower-order model correction, J. Comput. Phys., 327:225-244, 2016. 4.9

[27] J.M. Perez Jorda and W. Yang, A concise redefinition of the solid spherical harmonics and its use in fast multipole methods, J. Chem. Phys., 104:8003-8006, 1996. 4.9

[28] P. Kauf, Multi-scale approximation models for the Boltzmann equation, PhD Thesis, ETH Zürich, 2011. 4.1

[29] J. Koellermeier, Hyperbolic approximation of kinetic equations using quadrature-based projection methods, Master's Thesis, RWTH Aachen University, 2013. 4.9

[30] J. Koellermeier, R.P. Schaerer, and M. Torrilhon, A framework for hyperbolic approximation of kinetic equations using quadrature-based projection methods, Kinet. Relat. Models, 7(3):531549, 2014. 1, 3, 3.1, 4, 5

[31] J. Koellermeier and M. Torrilhon, Hyperbolic moment equations using quadrature-based projection methods, AIP Conf. Proc., 1628(1):626-633, 2014. 1

[32] J. Koellermeier and M. Torrilhon, Numerical solution of hyperbolic moment models for the Boltzmann equation, Eur. J. Mech. B Fluids, 64:41-46, 2017. 3.1

[33] J. Koellermeier and M. Torrilhon, Numerical study of partially conservative moment equations in kinetic theory, Commun. Comput. Phys., 21(4):981-1011, 2017. 1, 3, 3.1, A, A, A, A

[34] J. Koellermeier and M. Torrilhon, Simplified hyperbolic moment equations, in C. Klingenberg and M. Westdickenberg (eds.), Theory, Numerics and Applications of Hyperbolic Problems II, Springer Proceedings in Mathematics and Statistics, 2018. 1, 4.7, A, A, A, A.1, A.2

[35] J. Kowalski and M. Torrilhon, Moment approximations and model cascades for shallow flow, 
Commun. Comput. Phys., 25:669-702, 2019. 4.9

[36] Y. Kuang and H. Tang, Globally hyperbolic moment model of arbitrary order for one-dimensional special relativistic Boltzmann equation, J. Stat. Phys., 167(5):1303-1353, 2017. 1, 4.3

[37] C. D. Levermore, Moment closure hierarchies for kinetic theories, J. Stat. Phys., 83(5):1021-1065, 1996. 1

[38] G. Mascali and V. Romano, A non parabolic hydrodynamical subband model for semiconductors based on the maximum entropy principle, Math. Comput. Modelling, 55(3):1003-1020, 2012. 4.3

[39] M.M. Mizrahi, Generalized Hermite polynomials, J. Comput. Appl. Math., 1(3):137-140, 1975. B

[40] I. Müller and T. Ruggeri, Rational Extended Thermodynamics, Second Edition, Springer Tracts in Natural Philosophy, Springer-Verlag, New York, 37, 1998. 1

[41] G. Poette, B. Despres, and D. Lucor, Uncertainty quantification for systems of conservation laws, J. Comput. Phys., 228(7):2443-2467, 2009. 2

[42] H. Struchtrup and M. Torrilhon, Higher-order effects in rarefied channel flows, Phys. Rev. E, 78:046301, 2008. 3

[43] M. Torrilhon, Convergence study of moment approximations for boundary value problems of the Boltzmann-BGK equation, Commun. Comput. Phys., 18(3):529-557, 2015. 4.3

[44] F.M. White, Fluid Mechanics, McGraw-Hill International Editions. McGraw-Hill, 2003. 3

[45] D. Xiu and G. Karniadakis, Modeling uncertainty in steady state diffusion problems via generalized polynomial chaos, Comput. Meth. Appl. Mech. Eng., 191(43):4927-4948, 2002. 2

[46] W.A. Yong, Singular perturbations of first-order hyperbolic systems with stiff source terms, J. Diff. Eqs., 155(1):89-132, 1999. 3.2, A

[47] V.F. Zaitsev and A.D. Polyanin, Handbook of Exact Solutions for Ordinary Differential Equations, CRC Press, 2002. 2.1 